\title{
Coupled flow network and discrete element modeling of injection-induced crack propagation and coalescence in brittle rock
}

\author{
Guang Liu ${ }^{1,3} \cdot$ WaiChing Sun ${ }^{2}$ (D) Steven M. Lowinger ${ }^{2} \cdot$ ZhenHua Zhang $^{1} \cdot$ Ming Huang $^{1} \cdot$ \\ Jun Peng ${ }^{3,4}$
}

Received: 3 October 2017 / Accepted: 25 May 2018/ Published online: 9 June 2018

(c) Springer-Verlag GmbH Germany, part of Springer Nature 2018

\begin{abstract}
We present a numerical analysis on injection-induced crack propagation and coalescence in brittle rock. The DEM network coupling model in PFC is modified to capture the evolution of fracture geometry. An improved fluid flow model for fractured porous media is proposed and coupled with a bond-based DEM model to simulate the interactions among cracks induced by injecting fluid in two nearby flaws at identical injection rates. The material parameters are calibrated based on the macro-properties of Lac du Bonnet granite and KGD solution. A grain-based model, which generates larger grains from assembles of particles bonded together, is calibrated to identify the microscopic mechanical and hydraulic parameters of Lac du Bonnet granite such that the DEM model yields a ratio between the compressive and tensile strength consistent with experiments. The simulations of fluid injection reveal that the initial flaw direction plays a crucial role in crack interaction and coalescence pattern. When two initial flaws are aligned, cracks generally propagate faster. Some geometrical measures from graph theory are used to analyze the geometry and connectivity of the crack network. The results reveal that initial flaws in the same direction may lead to a well-connected crack network with higher global efficiency.
\end{abstract}

Keywords Brittle rock . Crack coalescence . Discrete element method $\cdot$ Flow network $\cdot$ Fluid-driven fracture

\section{Introduction}

Injecting fluid into aquifers is a multi-physical process occurring in many engineering applications, such as unconventional hydrocarbon recovery and $\mathrm{CO}_{2}$ sequestration [19, 29]. In particular, hydraulic fracturing in shale gas reservoirs is an important technique employed to enhance

WaiChing Sun

wsun@columbia.edu

1 School of Civil Engineering, Hefei University of Technology, Hefei, China

2 Department of Civil Engineering and Engineering Mechanics Technology, Columbia University, New York, USA

3 State Key Laboratory of Water Resources and Hydropower Engineering Science, Wuhan University, Wuhan, China

4 Key Laboratory of Rock Mechanics in Hydraulic Structural Engineering (Ministry of Education), Wuhan University, Wuhan, China hydrocarbon recovery in reservoirs of low permeability [16]. This technique has been adopted for a wide spectrum of applications, including gas or water well stimulation, energy extraction from geothermal reservoirs, and in situ stress measurement [10, 21, 58]. Hydraulic fracturing involves the injection of large volumes of fluid into subsurface formations to induce excess pore pressure. This excess pore pressure causes cracks to grow in the oilbearing or gas-bearing formations. The crack growth in return leads to higher effective permeability and therefore makes the extraction of hydrocarbon more feasible. Depending on the in situ stress state, the fluid-driven fracture may result in a complex fracture network growth detectable via micro-seismic monitoring [24].

In the past few decades, acoustic emission has provided important experimental data to understand the mechanism of the initiation and propagation of fluid-driven fractures $[2,27]$. For instance, Ishida et al. [33] studied how the permeability and texture of the host matrix, as well as the viscosity of the fluid, affect crack growth. They observed that 
cracks growth under excess oil pressure tends to be thicker and with fewer branches, while those cracks formed under excess water pressure tend to thinner and with more branches. Furthermore, the effective permeability is often dominated not only by the macroscopic porosity but also the micro-pores and localized features, such as cracks and joints.

These discontinuities may become a conduit when fluid is pressurized and injected into the wellbore. The fluid penetration and interfacial opening caused by the injection of pressurized fluid may lead to a reduction of fluid pressure and redistribution of stress around the propagating cracks. Furthermore, the viscous dissipation of fluid flow leads to a reduction of pressure as the distance increases from the source so that the fractures close to the fluid source tend to grow faster [79]. Understanding this twoway coupled hydromechanical effect of fluid-driven fracture is important for hydrocarbon recovery and the related geological applications, such as carbon dioxide storage $[14,22,39,59,65,68,73,74]$.

Rock formations may experience fracture extension and fluid flow infiltration when pressurized viscous fluid is poured into the deep fractured rock mass. Various theoretical models for hydraulic fracturing have been continuously developed in the past decades. For instance, Perkins and Kern [51] proposed the original Perkins and Kern (PK) model to describe the mechanics of hydraulic fracture based on the classic Sneddon elasticity plane strain crack solution. Nevertheless, the PK model neglected the fluid loss in the medium for convenience's sake, and the PK model may introduce non-negligible deviations in highly permeable formations. As a consequence, Nordgren [46] modified the PK model by incorporating the fracture opening in the lubrication equation. This change leads to the well-known Perkins-Kern-Nordgren (PKN) model (Fig. 1). The plane strain fracture propagating in an impermeable elastic rock mass has been studied in pioneering research done by Khristianovic and Zheltov [35] and Geertsma and De Klerk [30], which is later referred as Khristianovic-Geertsma-de Klerk (KGD) model (Fig. 1). In the KGD model, the fracture tip region plays an important role, due to the concentration of the fluid flow and the sharp pressure gradients in this region [20]. Both models, as shown in Fig. 1, provide important benchmarks for the verification of computational models. However, the PKN and KGD models only consider the propagation of a single fracture in an isotropic and homogeneous medium. Since a geological system is usually composed of anisotropic and heterogeneous media, the interaction and coalescence of fluid-driven fractures occurring during the life cycle of the reservoir are often predicted via computational models [31, 32, 47, 81].

In addition to the initiation and propagation of cracks, crack coalescence is an important phenomenon which may significantly alter the hydromechanical behaviors of brittle rocks. Crack coalescence in molded gypsum and Carrara marble specimens with preexisting open flaws has been studied at both macroscopic [77] and microscopic scales [78]. Using high-speed cameras to keep track of the crack

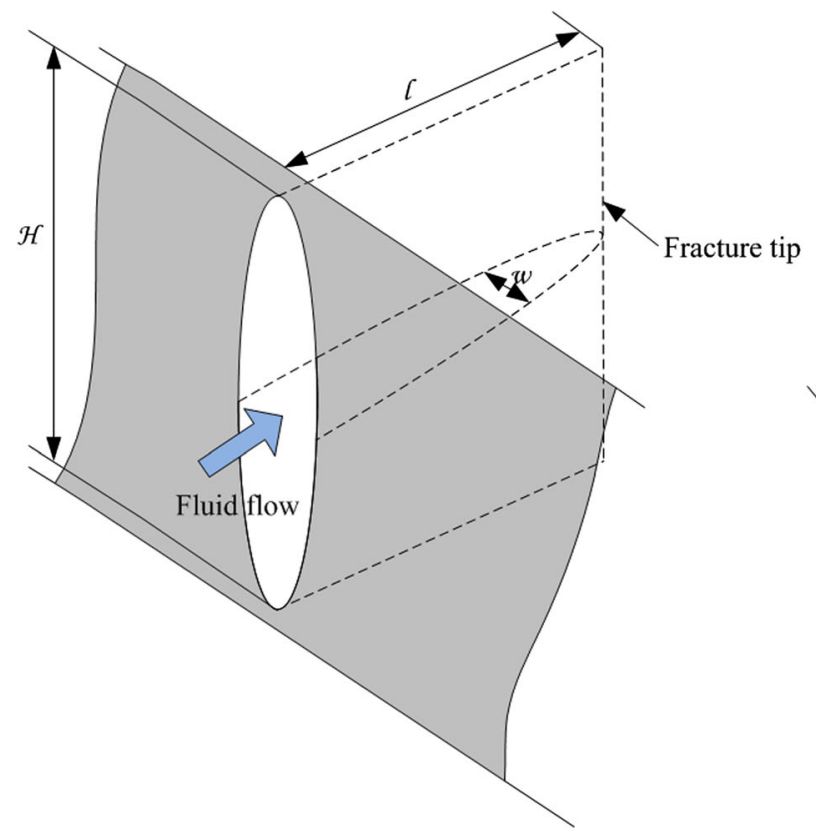

PKN fracture model

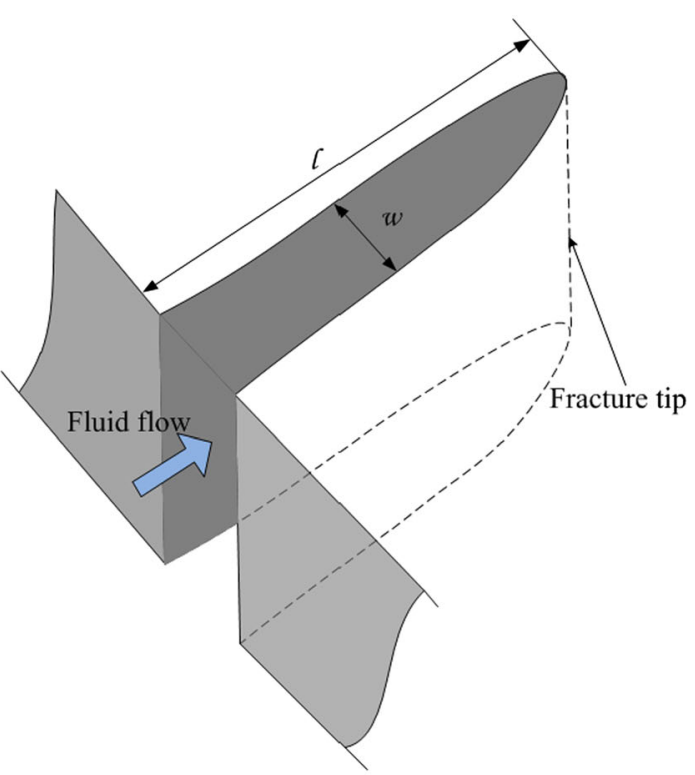

KGD fracture model

Fig. 1 Schematic illustration of PKN and KGD fracture model. After Khristianovic and Zheltov [35]; Perkins and Kern [51]; Geertsma and De Klerk [30] 
growth and coalescence, these experimental studies carefully analyzed the influences of flaw geometry, ligament length and flaw-pair distance on the likelihood of coalescence and the types of cracks involved in coalescence, bridging material, crack interaction, and energy release. Reyes and Einstein [55] characterized the pre-flaws in the sample as overlapping or non-overlapping, depending on the inclination of the rock bridge with respect to the direction of the applied load. They found that overlapping preexisting fractures coalesce through the interconnection of developing cracks and non-overlapping fractures coalesce through the secondary cracks [55]. According to experimental results of uniaxial and triaxial tests [48, 49], the crack coalescence pattern is determined by the inclination angle, length, and spacing of preexisting flaws in the specimen. However, these research results about crack coalescence do not involve the fluid injection-induced crack. Although significant progress has been made to characterize and model the interaction and coalescence of preexisting flaws and cracks, how fluid-driven cracks initiate, interact, and coalesce remains not well understood.

The objective of this paper is to use the pore-scale discrete hydromechanical simulations to analyze the mechanisms of the coalescence and interaction of cracks in porous media. Using a two-dimensional discrete element model coupled with a flow network, we simulate the hydromechanical coupling processes in brittle rock and analyze the mechanism of fracture propagation, interaction and coalescence driven by fluid injected at multiple flaws. Furthermore, we apply concepts from graph theory to examine the topological structure of the fluid and crack network during the process of fluid-driven fracture. Hydraulic fracturing is simulated using the discrete element method (DEM) code, and the main parameters in the model are calibrated from experimental data. Two initial flaws are configured in the model. Fluid is injected into the two flaws with same injection rates, and we observe the process of crack interaction and coalescence around the initial flaws. The influence of flaw orientation on crack geometry is illustrated by a series of simulations of hydraulic fracturing in hard rock. The concepts of shortest path and global efficiency from graph theory are used to analyze the evolutions of connectivities of the fluid flow and crack networks during the hydraulic fracturing.

\section{Methodology}

A two-dimensional particle flow code (PFC2D) is employed to perform numerical tests. In this section, the grain-scale discrete mechanics model at the contact points is first introduced. Then a modified fluid flow algorithm is described based on a pore network. The hydromechanical coupling scheme captures the grain-scale solid responses induced by fluid flow and the flow induced by fracture and solid deformation. These features allow one to simulate the crack propagation driven by fluid pressure, and the opening and closure of pore throats. The interactions between the solid and fluid phase are discussed in detail in the fluid flow algorithm. The hydrostatic pressure and viscous shear force are applied to solid particles as an unbalanced force in the simulation.

\subsection{Grain-scale discrete mechanics model}

Grains in a numerical specimen are represented by circular particles under two-dimensional plane strain condition. A discrete element model in PFC2D simulates the mechanical behavior of materials based on the balance of force and moment, as well as the force-displacement law at the contact point. The motion of circular particles obeys Newton's laws of motion, which can be written as

$\mathbf{F}_{\mathrm{i}}=-m\left(\mathbf{a}_{i}-\mathbf{g}_{i}\right)$

where $\mathbf{F}_{i}$ is the resultant force acting upon particle $i, m$ is the mass of a particle, $\mathbf{a}_{i}$ is the acceleration vector of particle $i$, and $\mathbf{g}_{i}$ is the body force acceleration vector (e.g., gravity loading).

In this research, the fluid drag force is applied to the solid grain as an unbalanced force. This drag force is updated incrementally and sequentially coupled with the mechanical solver. The resultant force acting upon particle $i\left(\mathbf{F}_{\mathrm{i}}\right)$ includes contact force from other particles, hydrostatic force summing up the pore pressure over the particle surface, and viscous shear force caused by viscous fluid flow [62]. The contact force from other solid grains is calculated in PFC automatically. The hydrostatic force is obtained from summing up the pore pressure over the particle surface [80]. The viscous shear force is inferred from a simplified parallelplate model. The details about the calculation of the pore pressure and shear force are discussed in Sect. 2.3.

The constitutive behavior of the contact is described by three characteristics at a contact point: stiffness, slip, and bond [34]. The stiffness relates the elastic contact force and relative displacement in the normal and tangential directions. As shown in Eq. (2), the normal component of the contact force, $F_{\mathrm{n}}$, is a function of the total normal displacements $U_{\mathrm{n}}$ and the contact normal stiffness $K_{\mathrm{n}}$ (unit Pa/ $\mathrm{m})$ in the contact normal direction. In the tangential direction, the stiffness relates the increment of the shear force $\Delta F_{\mathrm{s}}$ and the increment of shear displacement $\Delta U_{\mathrm{s}}$.

$\left\{\begin{array}{l}F_{\mathrm{n}}=K_{\mathrm{n}} U_{\mathrm{n}} \\ \Delta F_{\mathrm{s}}=-k_{\mathrm{s}} \Delta U_{\mathrm{s}}\end{array}\right.$

where $K_{\mathrm{n}}$ and $k_{\mathrm{s}}$ are the contact normal and tangential stiffnesses (unit $\mathrm{Pa} / \mathrm{m}$ ), respectively. In this paper, we adopt a linear contact model such that the elastic stiffnesses are 


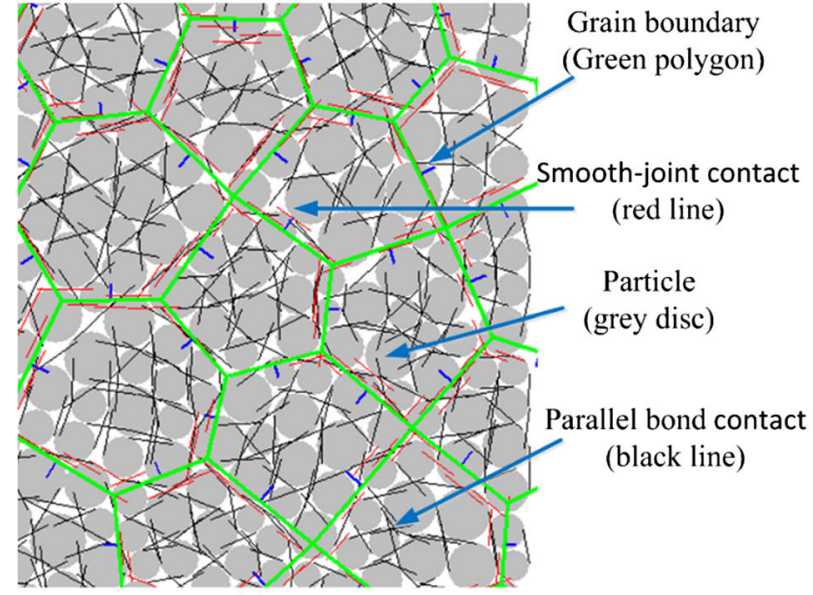

(a)
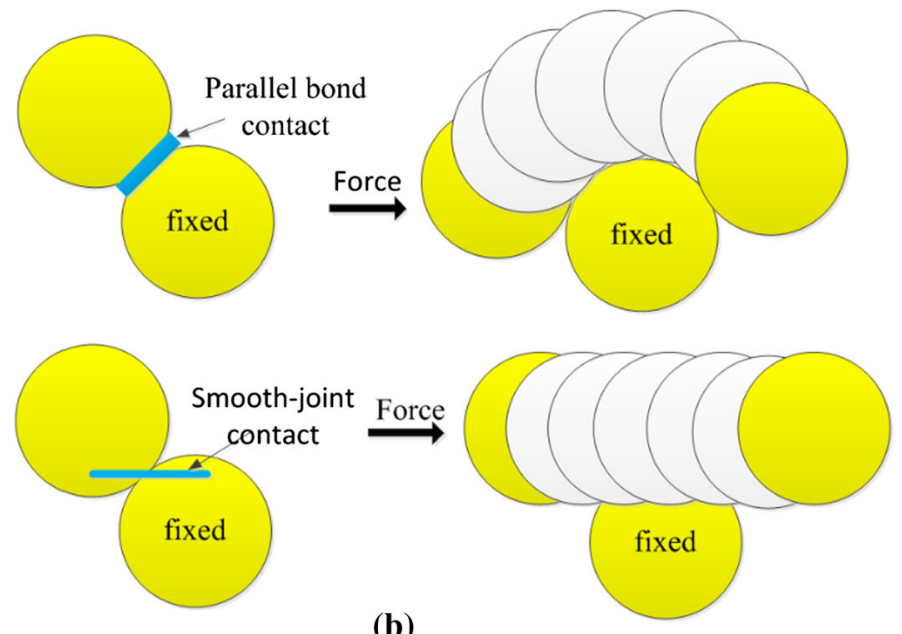

(b)

Fig. 2 Schematic diagram of grain-based model and different movement paths of particles for different contact models. a Grain-based model. The gray disks are particles in PFC. The polygonal meshes represent grain structure. b Movements of particles after breakage for different contact models. After Itasca Consulting Group [34]

independent of the displacement. Furthermore, a slip becomes perfectly plastic if the slip condition listed in Eq. (3) is met, i.e.,

$F_{\mathrm{s}}^{\max }=\mu\left|F_{\mathrm{n}}\right|$

where $F_{\mathrm{s}}^{\max }$ is the maximum allowable shear contact force and $\mu$ is the frictional coefficient. At each contact point, if $\left|F_{\mathrm{s}}\right|>F_{\mathrm{s}}^{\max }$, then slip will occur and the magnitude of $F_{\mathrm{s}}$ is set to $F_{\mathrm{s}}^{\mathrm{max}}$ in the next calculation cycle.

The bonded-particle model (BPM) is proposed to model constitutive responses of cohesive materials such as clayey soils and rocks [13, 34, 54]. The parallel and contact bond models are two distinct bonded-particle models available in the PFC. They are widely used to simulate the bonding behaviors between two grains. The parallel bond model acts as a pair of elastic springs with constant normal and shear stiffness at a finite cross section between two particles. Compared with the contact bond model, the parallel bond model is more suitable for rock. This is because the contact bond model may transmit both forces and moments at the contact point between particles and thus replicates the interaction mechanism of particles [56].

However, the assumptions of unbreakable grains and an idealization of circular (2D) and spherical particle used in BPM also make it difficult to replicate a realistic ratio between the tensile strength and the compressive strength of rock [52]. As the breakdown pressure of hydraulic fracturing depends on the tensile strength of rock, the conventional BPM simulations may overestimate the breakdown pressure in hydraulic fracture test. As a result, the grain-based model (GBM) proposed by Potyondy [52] is incorporated in our coupled discrete element model. GBM method mimics a synthetic material which consists of a large number of deformable and breakable polygonal grains cemented along their adjoining sides. In the grainbased model, each grain is formed by several bonded particles and the contacts of interface grains are depicted by smooth-joint model (Fig. 2). As shown in Fig. 2, each grain consists of several disks (gray), and these disks in a grain are bonded together with the parallel bond model. The grain boundary (the green mesh) has a low strength, and their contact property is assigned by smooth-joint contacts. The smooth-joint model mimics the behavior of an interface regardless of the local particle contact orientations along the user-specified interface. As shown in Fig. 2b, the balls of parallel bond move around each other after bond breakage, which can cause a local dilation. However, the balls located on the opposite sides of a smooth-joint contact are allowed to overlap and slide along the joint plane, which will not lead to a local dilation in the process of movement.

GBM method aims to capture the macro-mechanic properties by a lot of deformable and breakable grains. The DEM grains' positions are random, and they controlled by the initial disks packing. Note that the geometrical properties and positions of the grain structure have not been compared with those of real rock. However, the content for each mineral and the grain size in the numerical specimen are in accord with the laboratory data [25, 43].

Figure 3 illustrates the procedure to generate the grain assemblies. An initial disk packing is first generated according to the contents and grain size of each mineral in crystalline rocks. The polygonal grain structure is then generated by linking the internal-void centroids of the contact network from the initial disk packing [41, 52]. Afterward, an assembly of disks, which represents a rock 


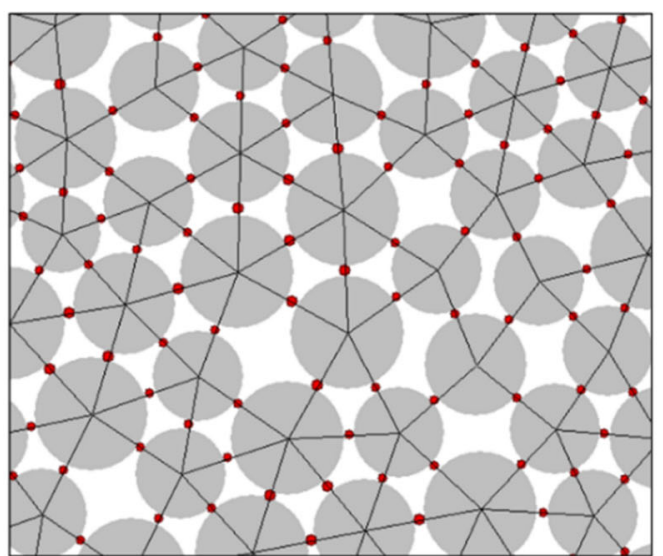

(a)

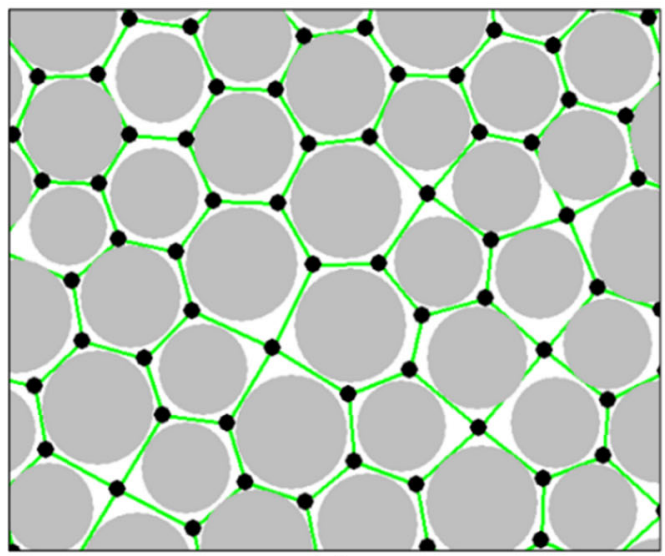

(c)

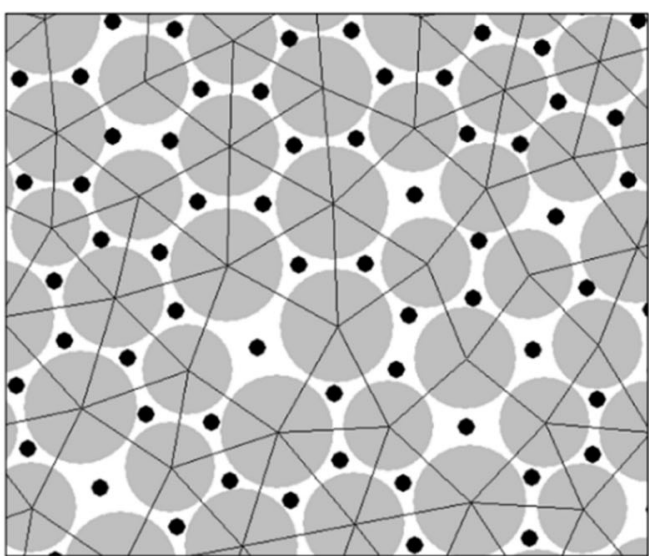

(b)

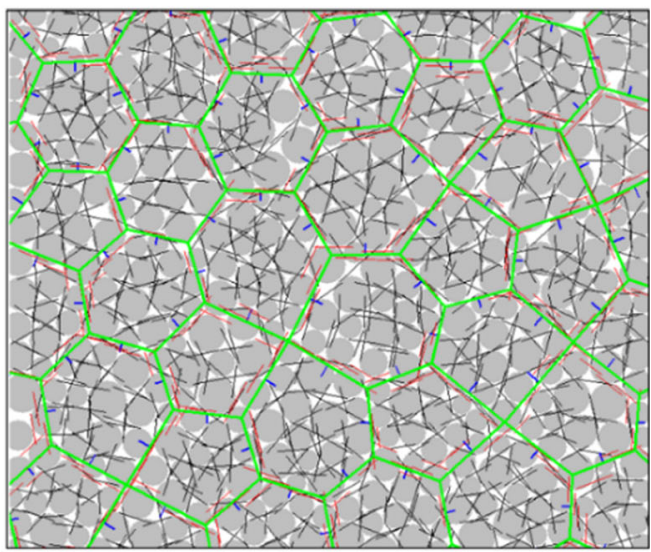

(d)

Fig. 3 Procedure to create a grain-based assembly of particles. a Connecting the centers of contacting disks from initial disks packing. The red points indicate the contact points. b Nodes of the grain structure. The black points are at the internal-void centroids. $\mathbf{c}$ Grain structure with nodes. The green polygonal mesh indicates the grain structure. d An assembly of particles is covered by the generated grain structure. The green network is the grain structure. The black lines between particles indicate parallel bond contacts, and the red lines along the grain structure are smooth-joint contacts. After Potyondy [52] (color figure online)

specimen, is overlaid by the generated polygonal grain structure. In the grain structure network, the contacts of disks along each edge of the grains are jointed together to form a smooth-joint contact that represents a grain boundary between two grains. The disks located in each polygonal mesh are bonded by parallel bond (a type of BPM) to form a mineral grain of crystalline rock (see Fig. 3d).

When either the magnitude of the tensile normal contact force or shear contact force exceeds the corresponding bond strength, the bond breaks and a micro-crack in PFC nucleates. In the 2D cases, a crack is represented by a line on the contact point that is perpendicular to the line between the centers of two particles. If the bond breaks due to the tensile force, the micro-crack is classified as the tensile crack. If the bond breaks due to shear force, the micro-crack is classified as shear crack.

\subsection{D fluid network identification}

The fluid flow algorithm that replicates pore fluid diffusion and the hydromechanical coupling effect was introduced in the computer code PFC by Cundall (unpublished technical note, 2000). Cundall's flow model creates a network that represents the topology of the void space and simulates the pore fluid transport in the representative network instead of the actual void space $[37,66,72]$. The solid skeleton in the flow model is idealized as an array of Voronoi polygons joined by elastic beams, which are subjected to tectonic stresses and the hydrostatic pressure of the pore fluid. This numerical treatment is feasible in two-dimensional space because the contact fabric and the void space can be viewed as a dual graph [61]. Therefore, by knowing the position and radius of all grains in the assembly, a flow network can be easily identified. 


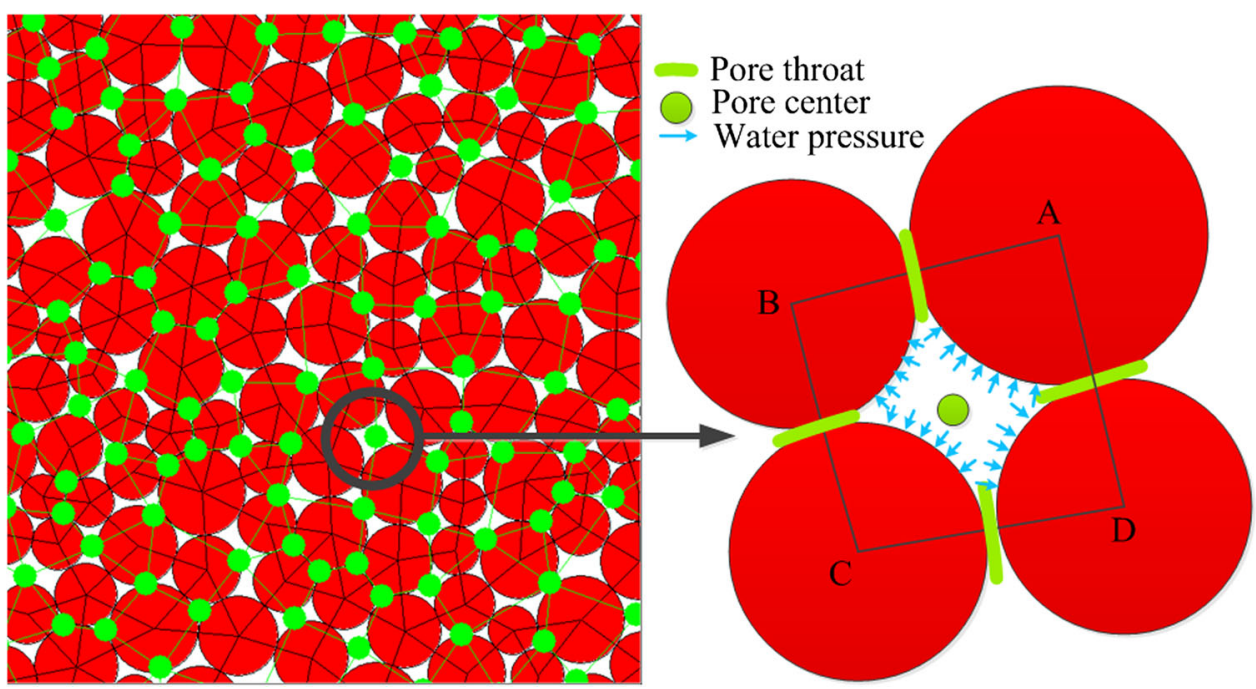

Fig. 4 Fluid network generation. The red circles are solid particles, the black meshes are pores, the green circles are pore centers, and the green lines are fluid channels between two pores (color figure online)

In the network-generating algorithm, each pore center surrounded by neighboring particles is denoted by a vertex, or node, while an edge is assigned to connect two nearby pore centers together (Fig. 4). This edge represents a flow channel in which the hydraulic conductivity is calculated based on the aperture. This fluid flow model is widely used to simulate fluid injection and hydraulic fracturing $[76,80]$.

Each void space in the flow network is generated by a series of neighboring particles connecting together $[3,62,80]$. Floating particles or particles with only one contact point will be neglected by the network generation algorithm. Instead, they form the occluded pore space. This algorithm simply selects the adjacent particles by checking contact point instead of measuring distance between particles. Thus, the algorithm will face a great challenge when a loose granular assembly is used. To circumvent these limitations, we introduce a distance-based contact criterion into the network generation algorithm such that the algorithm is now capable of handling loose granular assemblies. Before the identification of pore network, a contact criterion [Eq. (4)] is defined to estimate whether two particles are adjacent to each other.

$\sqrt{\left(x_{1}-x_{2}\right)^{2}+\left(y_{1}-y_{2}\right)^{2}} \leq c\left(R_{1}+R_{2}\right)$

where $x_{1}, x_{2}, y_{1}$, and $y_{2}$ are the coordinates of particles 1 and 2. $R_{1}$ and $R_{2}$ are the radii of particles 1 and 2 , respectively. $c$ is a parameter given by users to estimate the particle compactness. When $c=1$, this contact criterion based on distance degrade into the criterion based on the contact point. By using this new contact criterion, this network-generating algorithm can be extended to the loose granular assembly (see Fig. 5).

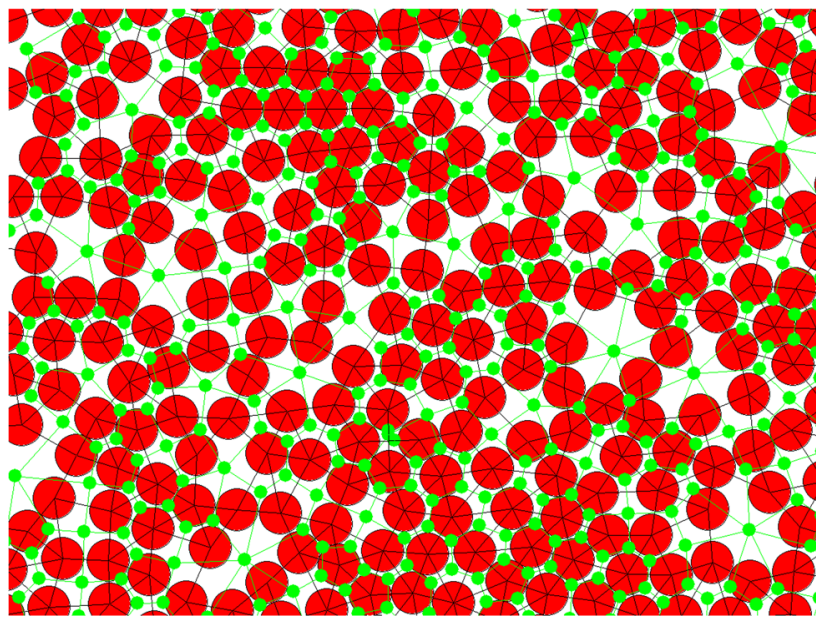

Fig. 5 A loose network extracted from the loose granular assembly. The parameter $c$ is 1.5 for this case

It should be noted that the two-dimensional pore network, in general, does not provide a realistic representation of 3D microstructures. For instance, Al-Raoush and Willson [5] compared different pore network generation techniques for unconsolidated porous media and found that two-dimensional networks do not provide a realistic representation of three-dimensional systems due to their inability to completely represent the pore connectivity. In this work, we use it as an analogical tool to replicate the pore connectivity variation brought by crack propagations.

\subsection{D fluid flow model}

The fluid flow model mainly involves the flux exchange process among different pores and the drag force applying 
to particles. As shown in Fig. 4, pore pressure is calculated at the vertices placed at the pore center, and the pore pressure gradient and Darcy's velocity are computed by comparing the pressure difference among the adjacent pore centers. In order to quantify the flow rate of the fluid, we model the space between the particles as a parallel-plate channel defined by two adjacent particles at their contact point with a unit depth (in the out-of-plane dimension). Therefore, the volumetric laminar flow rate $q$ (volume per unit time) in this pipe can be calculated by the cubic law [80]:

$q=\frac{a^{3} \Delta P}{12 \mu L}$

where $a$ is the aperture width of fluid channel which depends on the normal forces between the two particles, $\mu$ is the fluid viscosity, $\Delta P$ is the pressure difference between two pores, and $L$ is the length of the channel.

In this two-dimensional model, a unit out-of-plane thickness is assumed. An initial aperture is assumed to allow fluid flow through the intact contacts. The length of the channel, $L$, is often calculated using radii of the two particles $R_{1}$ and $R_{2}$ because the fluid channel is generated by the contact between particles [see Eqs. (6) and (7)] $[62,80]$. We identify the pore network by computing the distance between two particles, instead of considering the contact relation between two particles. Hence, we do not need to consider the number of contact points and we are capable of generating the pore network by non-contact particles. Given that a small gap may exist between two particles in a pore mesh (Fig. 4), we take the distance between two particle centers as the length of a pore channel.

$L=\frac{4 R_{1} R_{2}}{R_{1}+R_{2}}$

$L=R_{1}+R_{2}$

The aperture at the contact points is related to the contact force between two particles. If the normal force of the intact bond is compressive, the aperture $a$ can be expressed as

$a=\frac{a_{0} F_{0}}{F+F_{0}}$

where $a_{0}$ is an initial aperture which allows fluid flow when there is an overlap at the contact to simulate the permeability for intact rock. $F$ is the current normal compressive force at the contact points, and $F_{0}$ is the normal compressive force when the pipe aperture decreases to half of its initial aperture. Equation (8) implies that the aperture decreases with the increase in compressive force. Although previous research shows the aperture should decrease exponentially with the increase in compressive force [17],

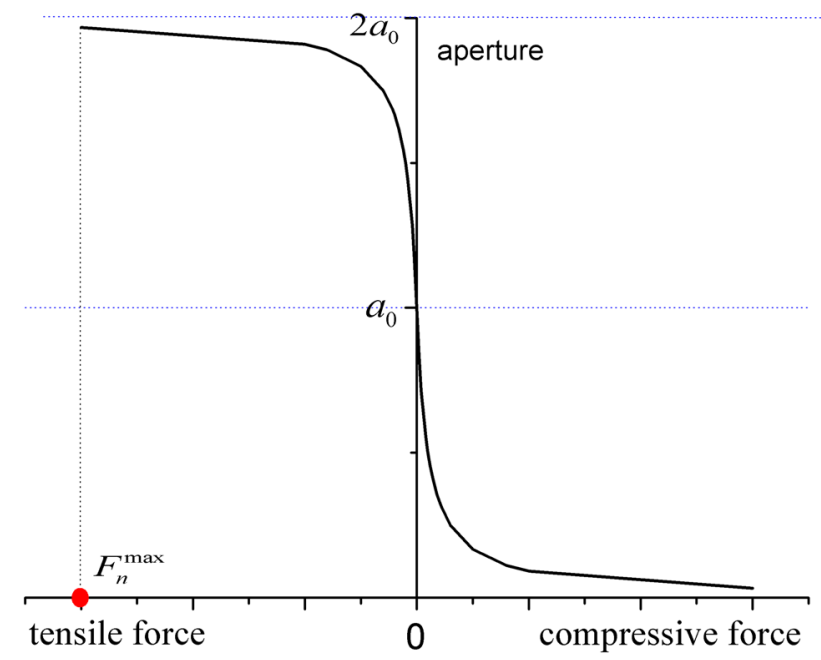

Fig. 6 Aperture variation with the contact force between two particles. This relation is only used before contact bond failure

Al-Busaidi [3] has shown that the simplification of Eq. (8) does not significantly alter the simulation results.

If the normal force at the contact point is tensile, Eq. (9) is introduced for aperture calculation in our simulation. In consequence, the hydromechanical effects can be embodied at the grain scale based on the computation of hydraulic aperture. Figure 6 presents the curve of aperture variation with the contact force. As a note, the relation in Fig. 6 only holds when the bond is undamaged. The maximum aperture will tend to $2 a_{0}$ in extreme tension state $\left(F_{\mathrm{n}}=F_{\mathrm{n}}^{\max }\right)$, and the aperture is $a_{0}$ at critical state from tension to compression. It is shown that two curves for tensile and compressive states are symmetric about point $\left(0, a_{0}\right)$.

$a=2 a_{0}-\frac{a_{0} F_{0}}{F_{0}-F}$

When the contact bond breaks, the crack aperture can be calculated by Eq. (10) [34].

$a=a_{c 0}+\lambda w$

where $w$ is the normal distance between the surfaces of the two particles, $\lambda$ is a dimensionless multiplier, and $a_{c 0}$ is the initial aperture for crack.

The contact failure under tensile stress may lead to crack growth. The permeability of the crack is observably higher than that in the rock matrix. For most models, the particles are much larger than the actual grains and therefore a multiplier $\lambda$ less than 1 is applied to make a realistic aperture width [3]. The normal distance between two particles' surfaces, $w$, is expressed by Eq. (11).

$w=d-R_{1}-R_{2}$

where $d$ is the distance between the two particle centers and $R_{1}, R_{2}$ are the radii of two particles. In each time step, fluid 
flow through the fluid channel causes a change of the fluid pressure in pores.

Considering a pore connecting $N$ fluid channels, the increment of the fluid pressure $\Delta P$, in a time step $\Delta t$, is written as

$\Delta P=\frac{K_{\mathrm{f}} \sum_{i=1}^{N} q_{i} \Delta t}{V_{d}}$

where $K_{\mathrm{f}}$ is the fluid bulk modulus, $V_{d}$ is the volume of the pore network, and $q_{i}$ is the flow rate of fluid channel $i$ connecting to this pore.

In this improved fluid coupled model, the area $S_{\mathrm{g}}$ with grid mark is taken as the volume of the pore network in two dimensions (Fig. 7). The area $S_{\mathrm{g}}$ can be calculated by subtracting the overlapping area of particles and the polygon $S_{\mathrm{o}}$, from the polygon area $S_{\mathrm{p}}$, formed by connecting the centers of surrounding particles [see Eq. (13)]. For each polygon, we can estimate its center position by average coordinates of vertexes. To obtain the area $S_{\mathrm{p}}$, the polygon can be divided into several triangles by connecting its center and vertexes. The area of polygon is the summation of all triangle areas for area calculation. In Fig. 7, the overlapping area $S_{\mathrm{o}}$ is the summation of four sectors formed by $\mathrm{ABCD}$ and four circles. Each sector area is estimated by the interior angle of polygon and circle radius.

Once a new crack is formed, the volume of the pore network must be updated to reflect the changes of pore geometry before the fluid step begins. Zhang et al. [80] explain that the variation of pore volume might be negligible if the topology of the fluid network remains unchanged and no crack has formed. In our simulations, the fracture geometry is continuously evolving. The appearance of a new crack will alter the position of the pores and augment the pore volume. However, it is computationally demanding to update the pore network with the crack propagation in each incremental step. It is also difficult to take into account of the evolving cracks in previous fluid coupled models available in PFC [3, 62, 76, 80]. In this improved fluid model, we consider the pore volume change induced by crack growth via Eq. (13). To avoid incrementally updating the connectivity of the pore network during the crack propagation, we assume that the crack growth will not alter the origin connectivity of the fluid network. Instead, it only imposes changes on the node positions of the network. As a result, the formation of a new crack may lead to the increase in pore volume by $m$ times. After a new crack is formed (Fig. 7), the pore volume $V_{d}$ is updated according to the crack number surrounding the pore center. This calculation method for a single pore volume $V_{d}$ in two dimensions can be written as $V_{d}=S_{\mathrm{g}}=\left\{\begin{array}{l}S_{\mathrm{p}}-S_{\mathrm{o}} \quad \text { no crack } \\ m^{n}\left(S_{\mathrm{p}}-S_{\mathrm{o}}\right) \quad n \text { cracks around a pore grid }\end{array}\right.$

where $S_{\mathrm{g}}$ is the grid marked area in Fig. 7, $S_{\mathrm{o}}$ is the overlapping area of particles and the polygon, $S_{\mathrm{p}}$ is the polygon area, $m$ is a constant which expresses the volume expansion induced by a single crack, and $n$ is the crack number surrounding this pore center.

In each fluid cycle, all fluid channels in the network are traversed and fluid transport driven by pressure in between two pore centers is captured via Eq. (5). Then the fluid pressure is updated by Eq. (12). The new fluid pressure in each pore center will be applied on the neighboring particles as an unbalanced force in the next mechanical cycle. In addition to this, the fluid pressure also contributes a normal component to the contact particles in our simulation, as a small gap is assumed on the particle contacts. The shear stress caused by fluid flow in the channel has been introduced by [62]. For this research, the shear stress is
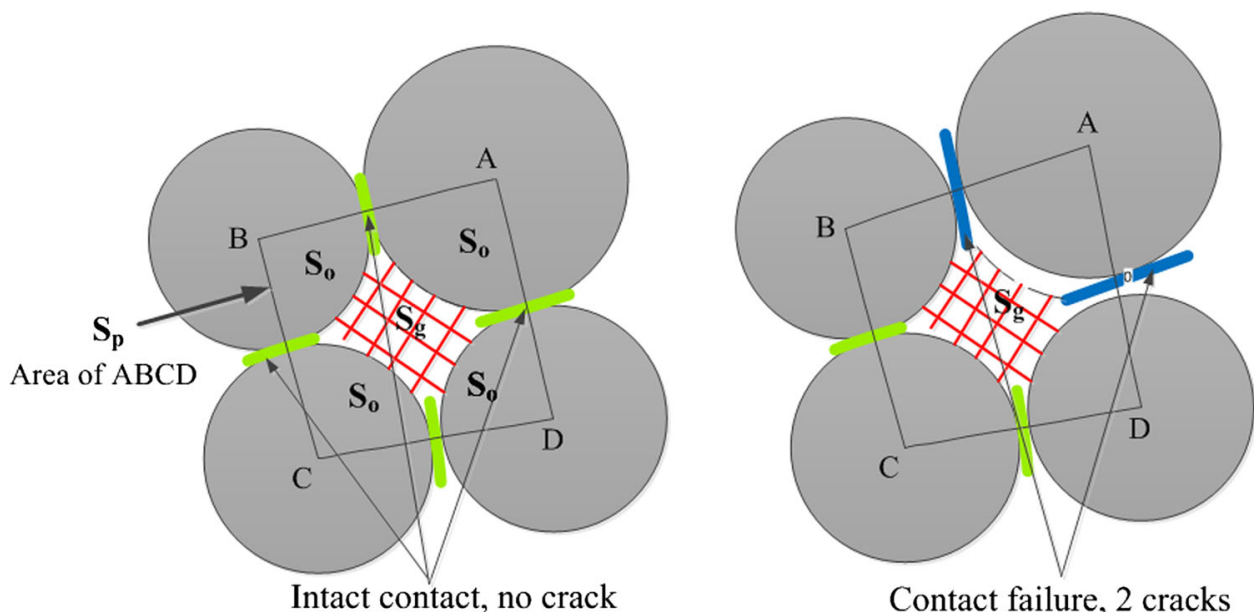

Fig. 7 Schematic diagram for the pore volume. The left figure shows a quadrangled mesh ABCD with four intact bonding contacts. The right figure shows the mesh $\mathrm{ABCD}$ with two cracks crossing. This is a simplified method to avoid updating the fluid network 


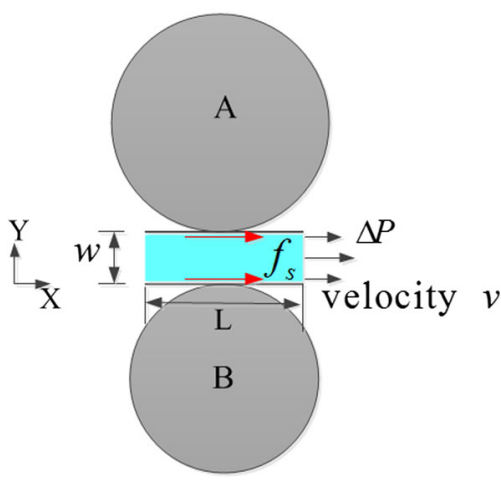

Fig. 8 Parallel-plate model for computing fluid shear stress. The width of the parallel plate depends on the contact force between two particles. The pressure difference is calculated according to the pressure of pore center on the two sides. After Shimizu et al. [62]

embodied using the same method. The fluid transport through the contact point is described by the parallel-plate model (Fig. 8). Consequently, the fluid velocity $v$ for laminar flow between two parallel plates can be expressed as

$v=\frac{w^{2} \Delta P}{2 \mu L}\left(\frac{y}{w}-\frac{y^{2}}{w^{2}}\right)$

Therefore, the shear stress caused by viscous fluid flow is given by Eq. (15). Taking $y=0$ and assuming $L$ as the length of the parallel plate, the total fluid shear force acting on the particles is $f_{\mathrm{s}}=\frac{w \Delta P}{2}$.

$\tau=\mu \frac{d v}{d y}=\left(\frac{w}{2}-y\right) \frac{\Delta P}{L}$

\subsection{Numerical stability and efficiency improvement}

In this section, we discuss the numerical stability and attempt to promote the computation speed by increasing the critical time step with a new strategy. The time step for mechanical cycle is determined automatically by PFC. It should be noted that the time step discussed here is used for fluid calculation. The fluid calculation launches after mechanical cycle for solid grains. Considering a fluid channel connected to pore ABCD (see Fig. 4), the influx $q_{1}$ caused by the pressure difference $\Delta P^{\prime}$ in a time step can be expressed as

$q_{1}=\frac{a^{3} \Delta P^{\prime}}{12 \mu L}$

Assuming that $\mathrm{N}$ fluid channels connect to this pore, the influx $q$ in this pore caused by the pressure perturbation in a time step is simplified as
$q=N q_{1}=\frac{N a^{3} \Delta P^{\prime}}{12 \mu L}$

The fluid flow will cause a pressure response $\Delta P^{\prime \prime}$ by Eq. (12).

$\Delta P^{\prime \prime}=\frac{K_{\mathrm{f}} q \Delta t}{V_{d}}$

For stability, the pressure response $\Delta P^{\prime \prime}$ must be less than the original pressure difference $\Delta P^{\prime}[34,80]$. Let pressure response $\Delta P^{\prime \prime}$ equal to pressure difference $\Delta P^{\prime}$. Combining Eqs. (17) and (18), we can obtain the critical time step $\Delta t_{c}$ for the fluid calculation.

$\Delta t_{c}=\frac{12 \mu L V_{d}}{N a^{3} K_{f}}$

Generally, the actual time step should be less than $\Delta t_{c}$ for numerical stability. However, due to the great difference of aperture between rock matrix and crack, the critical time step used in the fluid cycle for the rock matrix, denoted by $\Delta t_{c m}$ is much bigger than that for the crack, denoted by $\Delta t_{c c}$. To maintain the numerical stability, one has to choose a very small time step [62,80], at least $\Delta t_{c c}$. This choice may nevertheless increase the computation time significantly.

In our simulations, a new strategy is employed to increase the critical time step in the fluid cycle without losing numerical stability. Assume that we still adopt the time step, $\Delta t_{c m}$ in the calculation. Undoubtedly, this will cause numerical instability in the area where cracks appear, as this area needs time step reducing to $\Delta t_{c c}$ to maintain stability. However, we may analyze the final state of water pressure in arbitrary connected pores 1 and 2 in this area. Assume that a fluid channel connects to pores 1 and 2 (Fig. 9). The pore volumes for pores 1 and 2 are $V_{d 1}$ and $V_{d 2}$, respectively, and the volume increments of fluid in pores 1 and 2 are $\Delta V_{\mathrm{f} 1}$ and $\Delta V_{\mathrm{f} 2}$, respectively. The fluid pressures in pores 1 and 2 are $P_{1}$ and $P_{2}$, respectively. Without loss of generality, let $P_{1} \geq P_{2} . P_{1}$ and $P_{2}$ can be given by

$\left\{\begin{array}{l}P_{1}=\frac{\Delta V_{\mathrm{f} 1} K_{\mathrm{f}}}{V_{d 1}} \\ P_{2}=\frac{\Delta V_{\mathrm{f} 2} K_{\mathrm{f}}}{V_{d 2}}\end{array}\right.$

Over time, the pressure difference between the two pore centers would progressively reduce until the flow reaches the steady state. Since the pressure changes are related to the fluid volume changes, we need to obtain the fluid volume increment in order to update the pore pressure. The fluid volume increment is given by $\Delta V=q \Delta t$ in the simulation. However, the fluid volume increment in a time step should be less than the ultimate fluid volume increment 


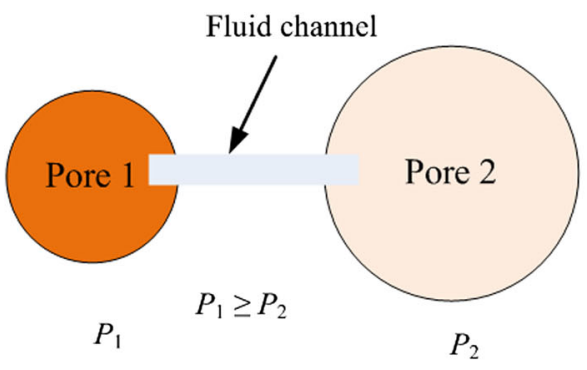

Initial state

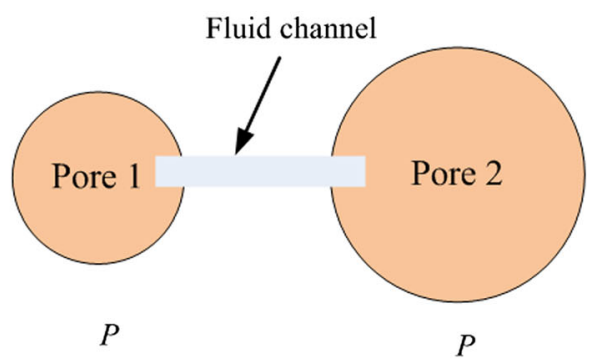

Final state

Fig. 9 Schematic diagram for the pressure response caused by fluid flow in a single fluid channel. The fluid pressures in pores 1 and 2 are P1 and $\mathrm{P} 2$, respectively. The left figure is the initial state for pores 1 and 2 . The right figure is the final state for pores 1 and 2

$\Delta V_{\mathrm{f}}$ such that the pressure will stabilize at $\mathrm{P}$ after enough time (Fig. 9). We attempt to deduce ultimate fluid volume increment $\Delta V_{\mathrm{f}}$ from the initial state to final state. If the calculated $\Delta V=q \Delta t$ is greater than $\Delta V_{\mathrm{f}}$, the fluid volume increment is set to $\Delta V_{\mathrm{f}}$ in our algorithm.

Due to $\Delta t_{c m} \gg \Delta t_{c c}$, the fluid pressure in the crack area will stabilize at $P$ if we use $\Delta t_{c m}$ as the time step. The final fluid pressure $P$ can be obtained by

$P=\frac{\left(\Delta V_{\mathrm{f} 1}+\Delta V_{\mathrm{f} 2}\right) K_{\mathrm{f}}}{V_{d 1}+V_{d 2}}=\frac{P_{1} V_{d 1}+P_{2} V_{d 2}}{V_{d 1}+V_{d 2}}$

Therefore, the ultimate fluid pressure variations in pores 1 and 2 are expressed as

$$
\left\{\begin{array}{l}
\Delta P_{u 1}=P_{1}-P=\frac{V_{d 2}\left(P_{1}-P_{2}\right)}{V_{d 1}+V_{d 2}} \\
\Delta P_{u 2}=P-P_{2}=\frac{V_{d 1}\left(P_{1}-P_{2}\right)}{V_{d 1}+V_{d 2}}
\end{array}\right.
$$

According to Eq. (12) (taking $N=1, \Delta V=q t$ ), the ultimate fluid volume increments, $\Delta V_{u 1}, \Delta V_{u 2}$ in pores 1 and 2 are derived by

$$
\left\{\begin{array}{l}
\Delta V_{u 1}=\Delta P_{u 1} \frac{V_{d 1}}{K_{f}}=\frac{V_{d 1} V_{d 2}\left(P_{1}-P_{2}\right)}{K_{f}\left(V_{d 1}+V_{d 2}\right)} \\
\Delta V_{u 2}=\Delta P_{u 2} \frac{V_{d 2}}{K_{f}}=\frac{V_{d 1} V_{d 2}\left(P_{1}-P_{2}\right)}{K_{f}\left(V_{d 1}+V_{d 2}\right)}
\end{array}\right.
$$

From Eq. (23), we have $\Delta V_{u 1}=\Delta V_{u 2}$, in which the continuity condition for fluid is satisfied. After long enough time, the pressure in two pores will reach the final state and the fluid volume increment is $\Delta V_{u 1}=\Delta V_{u 2}$. The above derivation process only considers the fluid flow from pore 1 to pore 2 . In the actual pore network, a pore may be connected to several other pores. This causes the real ultimate fluid volume increment $\Delta V_{u}$ to be smaller than $\Delta V_{u 1}$ and $\Delta V_{u 2}$. Therefore, a safety factor $\alpha(0<\alpha<1)$ is introduced to estimate the real ultimate fluid volume increment $\Delta V_{u}$.

$\Delta V_{u}=\alpha \Delta V_{u 1}=\alpha \frac{V_{d 1} V_{d 2}\left(P_{1}-P_{2}\right)}{K_{f}\left(V_{d 1}+V_{d 2}\right)}$
As a consequence, in a given fluid cycle with time step $\Delta t_{c m}$, if the fluid volume increment $\Delta V$ calculated by Eq. (12) $\left(\Delta V=q \Delta t_{c m}\right)$ is greater than $\Delta V_{u}$, that means the fluid transport by this channel will reach the final state. That is to say, the pore pressure in the two ends of the fluid channel will reach the pressure in Eq. (21), and the fluid increment is $\Delta V_{u}$. This provides a strategy to calculate the fluid increment $\Delta V_{u}$ (Eq. (24)) when we use $\Delta t_{c m}$ as the critical time step. Therefore, according to the illustration above, the time step $\Delta t_{c m}$ still can be used as the critical time step as long as the ultimate fluid volume increment is decided by Eq. (24). This method will enhance the

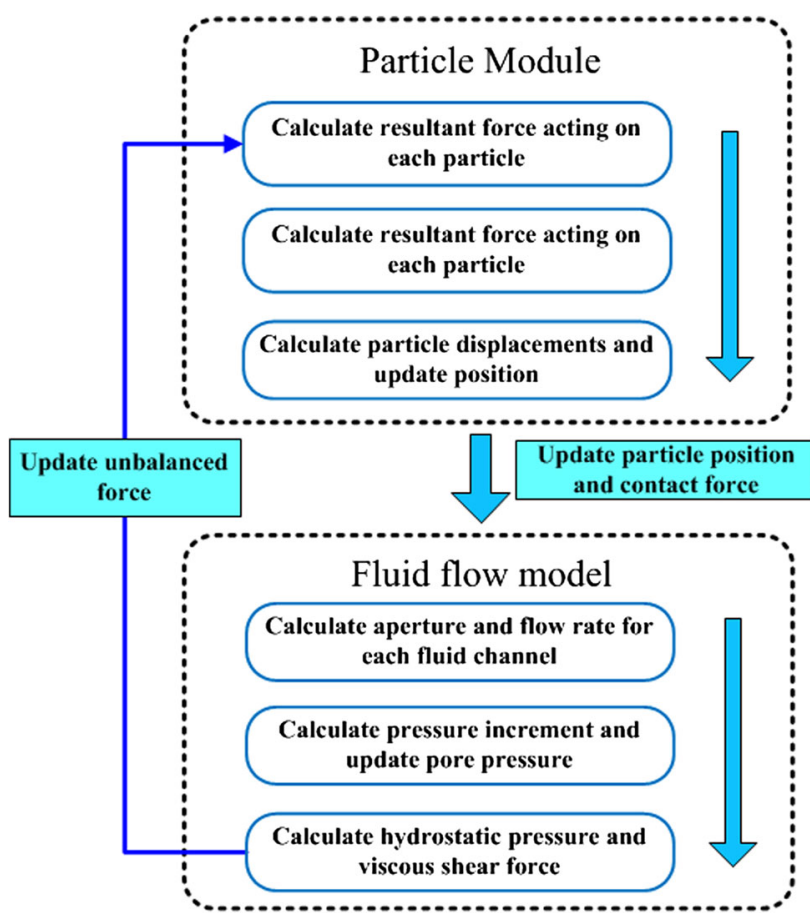

Fig. 10 Schematic of calculation sequences in the simulation. The particle module is provided by PFC and its time step is determined by PFC automatically. The fluid flow model is implemented using FISH language in $\mathrm{PFC}$ 
computational efficiency significantly when cracks grow in the specimen.

Figure 10 presents a flow chart of the calculation process. The built-in particle module of PFC is used to update the particle position and contact force. The fluid flow model is used to calculate the drag force applying on each particle. The drag force is applied as an unbalanced force in the mechanical cycle in particle module. The time step in mechanical cycle is determined by PFC automatically. In the fluid model, a fix time step is adopted. This time step should less than the critical time step to maintain numerical stability [34].

\section{Numerical specimen generation and material parameter calibration}

In this section, the grain-based specimens are generated to simulate the macro-mechanical behavior of rock. The model parameters are calibrated by the results of a laboratory experiment performed on Lac du Bonnet granite $[43,63]$.

\subsection{Numerical rock specimen using the grain- based model}

According to the research by Eberhardt et al. [25], Lac du Bonnet granite mainly consists of four minerals: k-feldspar, plagioclase, quartz, and biotite. For Lac du Bonnet gray granite, the content of the four minerals, k-feldspar, plagioclase, quartz, and biotite, is $45,20,30$, and $5 \%$, respectively. Based on the content of minerals, a numerical

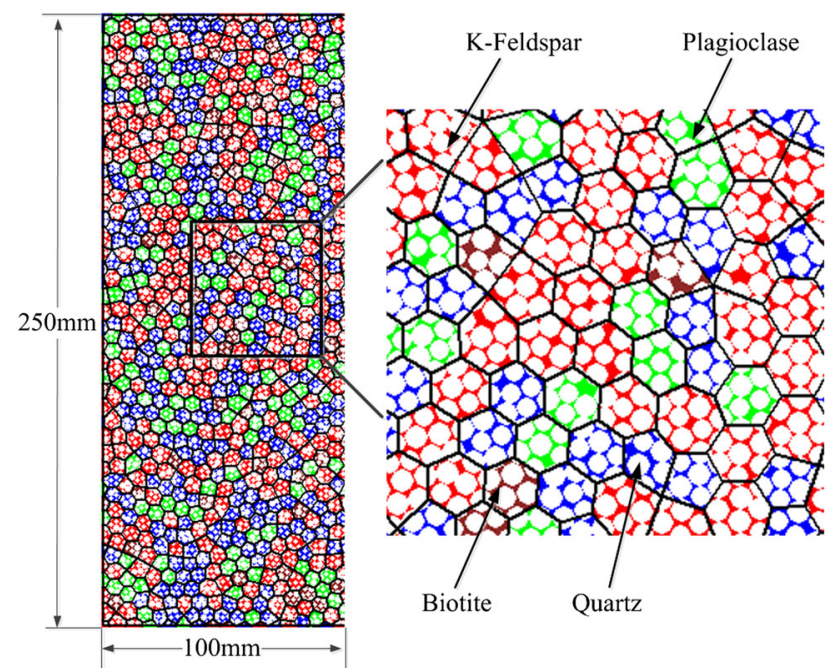

Fig. 11 A grain-based numerical specimen of granite. The red, green, blue, and brown areas indicate k-feldspar, plagioclase, quartz, and biotite, respectively (color figure online) specimen is generated to conduct the uniaxial compression test.

Figure 11 presents a numerical specimen created by grain-based model. The synthetic rock specimen has a height of $250 \mathrm{~mm}$ and width of $100 \mathrm{~mm}$. The ratio of height and to width is 2.5 , which is in accordance with the experimental sample specimen shape reported by Martin [43]. The black mesh in Fig. 11 shows the grain structure. Note that the topological and statistical properties of the grain structure have not been compared with those of Lac $\mathrm{du}$ Bonnet granite. According to the statistical data by Martin [43], the grain size of Lac du Bonnet granite ranges from 3 to $9 \mathrm{~mm}$. In this research, all the grain radii are $3.0 \mathrm{~mm}$. According to the research by Peng et al. [50], the grain size difference can cause the micro-geometric heterogeneity of grain structure. The reason we use the same grain radii is that we want to eliminate the influence of micro-geometric heterogeneity on the crack pattern. Each grain consists of several cemented particles, and grains are deformable and breakable. The numerical specimen includes four minerals. The modeling parameters of four minerals are different, and these parameters are calibrated by numerical uniaxial compression and direct tension tests until the macro-mechanical properties captured by the numerical tests match the corresponding properties obtained from the laboratory test.

\subsection{Material parameters calibration}

Because microscopic mechanical parameters cannot be obtained directly from macroscopic laboratory experiments, a numerical calibration is required to determine these microscopic parameters [36, 40, 56]. A uniaxial compression test is performed to compare to the mechanical response from the laboratory experiment for Lac du Bonnet granite [27]. Figure 12 shows the contact force chain of grain boundary and the distribution of microcracks at post-peak stage, in which axial stress is 0.8 times of peak strength. It is found that the compressive force chains distribute in strips along the direction of axial compression. As seen in Fig. 12b, a lot of microscopic tensile cracks (blue) propagate along the grain boundaries. A small number of tensile cracks (black) cross the grains to form the intra-grain cracks.

Table 1 presents a comparison of macro-mechanical properties between an experiment and the corresponding numerical simulation. In this simulation, the tensile strength is inferred from an uniaxial tension test. The Young's modulus $E$ and Poisson's ratio $v$ are computed by assuming plane strain condition and using the stress and strain increments occurring between the start of the test and the point that marks one-half of the peak stress [53]. As shown in Table 1, the macro-mechanical properties, 


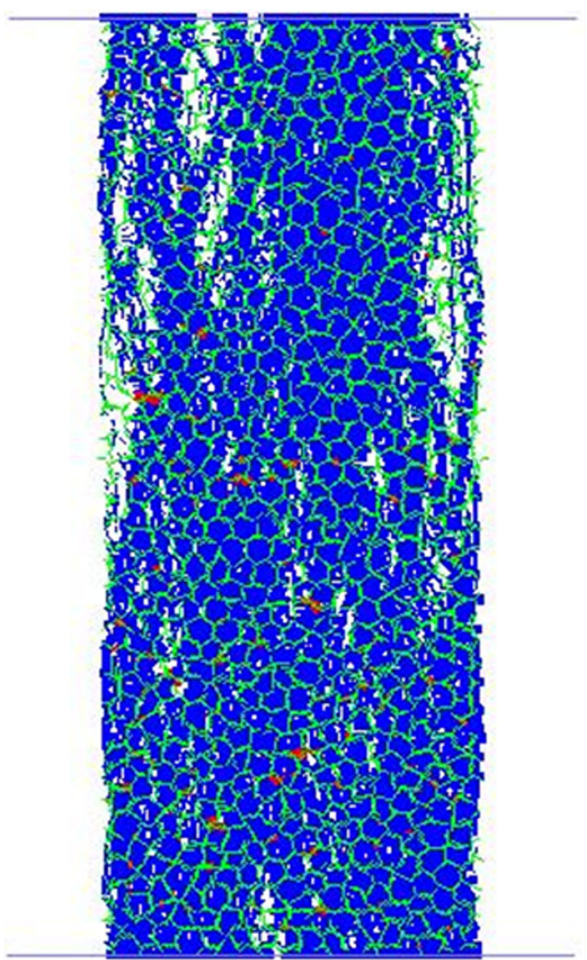

(a)

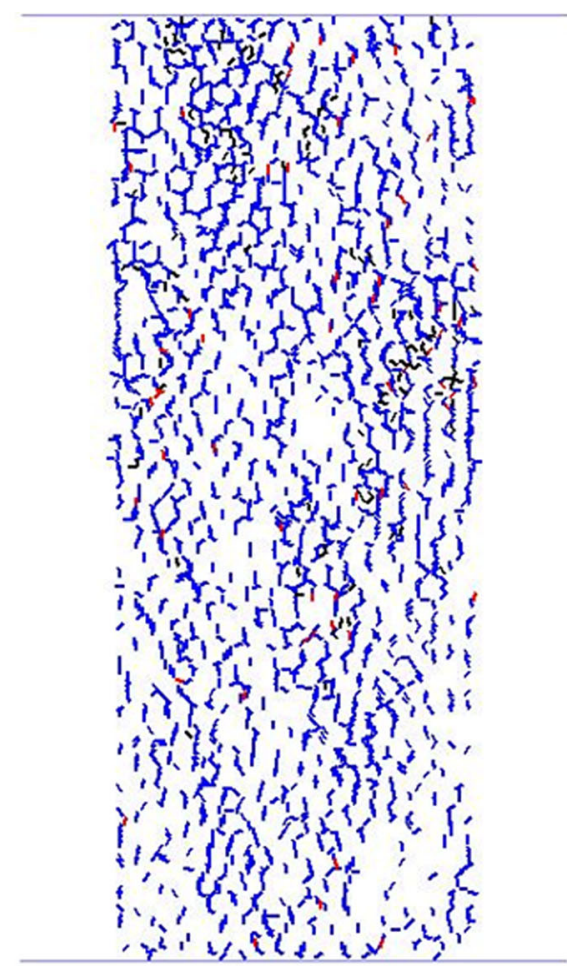

(b)

Fig. 12 a Contact force chain of grain boundaries after the test. Blue area is compressive and red area is tensile. The grain boundary is represented by the green polygons. b Distribution of micro-cracks. The blue lines represent inter-grain tensile cracks, the red lines represent intergrain shear cracks, and the black lines represent intra-grain tensile cracks (color figure online)

Table 1 Comparison of macro-mechanical properties of Lac du Bonnet granite $^{\mathrm{a}}$

\begin{tabular}{lcl}
\hline Macro-mechanical properties & Experiment & PFC-GBM \\
\hline Young's modulus $E(\mathrm{GPa})$ & $69 \pm 5.8$ & 66.2 \\
Poisson's ratio $v$ & $0.26 \pm 0.04$ & 0.24 \\
Uniaxial compressive strength $\sigma_{\mathrm{c}}(\mathrm{MPa})$ & $200 \pm 22$ & 203.6 \\
Tensile strength $\sigma_{\mathrm{t}}(\mathrm{MPa})$ & $9.3 \pm 1.3$ & 9.3 \\
Ratio of compression to tension, $\sigma_{\mathrm{c}} / \sigma_{\mathrm{t}}$ & 21.5 & 21.9 \\
\hline
\end{tabular}

${ }^{a}$ The experiment samples from the URL were obtained from shallow depth $(0-200 \mathrm{~m})$ [43]

especially the tensile strength, can be replicated by the grain-based model. The macro-mechanical properties, such as Young's modulus E, Poisson's ratio $v$, uniaxial compressive strength (UCS) $\sigma_{\mathrm{c}}$, tensile strength $\sigma_{\mathrm{t}}$ and the ratio of unconfined-compressive strength to tensile strength $\sigma_{\mathrm{c}} / \sigma_{\mathrm{t}}$, which are calculated by simulation fall in the range given by Martin [43]. The ratio of the unconfined-compressive strength to tensile strength is 21.9 , which is very close to the mean experiment value of 21.5. The ratio for the BPM model consisting of parallel-bonded disks is often too low to match actual value [52]; however, the calculated value using PFC-GBM agrees well with the actual value in our numerical model. Since the calculated macro-mechanical properties (Table 1) match that from experiment, the micro-parameters listed in Table 2 are used for modeling the mechanical response of Lac du Bonnet granite.

The calibration of hydraulic parameters mainly involves the determination of initial aperture $a_{0}, a_{c 0}$, multiplier $\lambda$, and constant $m$. The appropriate hydraulic parameters should be chosen so that the corresponding macro-permeability of Lac du Bonnet granite in the simulation matches the experimental measurement in previous literature [63]. Al-Busaidi [3] suggested using following equation to estimate the macro-permeability of numerical model.

$k_{p}=\frac{1}{12 V} \sum_{i=1}^{N} L_{i} w_{i}^{3}$

where $k_{\mathrm{p}}$ is the macro-permeability of numerical model, $V$ is the volume of the specimen, $L_{i}$ is the length of fluid channel $i, w_{i}$ is the aperture of fluid channel $i$, and $N$ is the total number of fluid channels.

The macro-permeability calculated via Eq. (25) is $1.02 \times 10^{-17} \mathrm{~m}^{2}$. This magnitude of effective permeability is within the range of given by Souley et al. [63]. The 
Table 2 Calibrated modeling parameters for numerical specimen of Lac du Bonnet granite

\begin{tabular}{|c|c|c|c|c|}
\hline \multirow[t]{2}{*}{ Micro-parameters } & \multicolumn{4}{|l|}{ Value } \\
\hline & $\begin{array}{l}\text { K- } \\
\text { Feldspar }\end{array}$ & Plagioclase & Quartz & Biotite \\
\hline Particle density $\left(\mathrm{kg} / \mathrm{m}^{3}\right)$ & 2560 & 2630 & 2650 & 3050 \\
\hline $\begin{array}{l}\text { Normal to shear stiffness } \\
\text { ratio of particles }\end{array}$ & 1.5 & 1.5 & 1.5 & 1.5 \\
\hline $\begin{array}{l}\text { Contact modulus of } \\
\text { particles }(\mathrm{GPa})\end{array}$ & 58.0 & 60.0 & 65.0 & 55.0 \\
\hline $\begin{array}{l}\text { Parallel bond modulus } \\
\quad(\mathrm{GPa})\end{array}$ & 46.0 & 52.0 & 55.0 & 40.0 \\
\hline $\begin{array}{l}\text { Normal to shear stiffness } \\
\text { ratio of bond }\end{array}$ & 1.5 & 1.5 & 1.5 & 1.5 \\
\hline $\begin{array}{l}\text { Parallel bond tensile } \\
\text { strength (MPa) }\end{array}$ & 360 & 380 & 350 & 320 \\
\hline $\begin{array}{l}\text { Cohesion of parallel bond } \\
(\mathrm{MPa})\end{array}$ & 320 & 350 & 380 & 300 \\
\hline $\begin{array}{l}\text { Friction coefficient of } \\
\text { particles }\end{array}$ & 1.0 & 1.0 & 1.0 & 1.0 \\
\hline $\begin{array}{l}\text { Parallel bond friction } \\
\text { angle }\left({ }^{\circ}\right)\end{array}$ & 50.0 & 51.0 & 52.0 & 48.0 \\
\hline \multicolumn{5}{|c|}{ Micro-properties of grain boundaries (Smooth-joint contacts) } \\
\hline \multicolumn{2}{|l|}{$\begin{array}{l}\text { Smooth-joint contact normal } \\
\text { stiffness factor }\end{array}$} & \multicolumn{3}{|l|}{0.65} \\
\hline \multicolumn{2}{|c|}{$\begin{array}{l}\text { Smooth-joint shear normal stiffness } \\
\text { factor }\end{array}$} & \multicolumn{3}{|l|}{0.65} \\
\hline \multicolumn{2}{|c|}{$\begin{array}{l}\text { Smooth-joint bond tensile strength } \\
(\mathrm{MPa})\end{array}$} & \multicolumn{3}{|l|}{12.0} \\
\hline \multicolumn{2}{|c|}{ Smooth-joint bond cohesion (MPa) } & \multicolumn{3}{|l|}{165.0} \\
\hline \multicolumn{2}{|l|}{$\begin{array}{l}\text { Smooth-joint bond friction } \\
\text { coefficient }\end{array}$} & \multicolumn{3}{|l|}{1.5} \\
\hline \multicolumn{2}{|c|}{ Smooth-joint bond friction angle $\left(^{\circ}\right)$} & \multicolumn{3}{|l|}{42.0} \\
\hline \multicolumn{5}{|l|}{ Hydraulic parameters } \\
\hline \multicolumn{2}{|c|}{$\begin{array}{l}\text { Initial aperture for intact bond, } a_{0} \\
(\mathrm{~m})\end{array}$} & \multicolumn{3}{|l|}{$5.0 \times 10^{-7}$} \\
\hline \multicolumn{2}{|c|}{$\begin{array}{l}\text { Initial aperture for failure bond, } a_{c 0} \\
(\mathrm{~m})\end{array}$} & \multicolumn{3}{|l|}{$1.25 \times 10^{-4}$} \\
\hline \multicolumn{2}{|l|}{ Viscosity, $\mu(\mathrm{P}$ a $\cdot \mathrm{s})$} & \multicolumn{3}{|l|}{$1.0 \times 10^{-3}$} \\
\hline \multicolumn{2}{|c|}{ Bulk modulus of fluid, $K_{f}(\mathrm{GPa})$} & \multicolumn{3}{|l|}{6.5} \\
\hline \multicolumn{2}{|l|}{ Multiplier, $\lambda$} & \multicolumn{3}{|l|}{0.2} \\
\hline \multicolumn{2}{|l|}{ Constant, $m$} & \multicolumn{3}{|l|}{1.2} \\
\hline
\end{tabular}

corresponding initial aperture and viscosity are listed in the above table. The dimensionless multiplier $\lambda$ is 0.2 , and the bulk modulus of the fluid is $6.5 \mathrm{GPa}$. The initial aperture for failure bond, $a_{c 0}$, and constant, $m$, is determined by KGD hydraulic fracture model, which is given in the following discussion.

The KGD hydraulic fracture model $[30,35]$ is adopted to examine the validity of numerical model. The KGD model considers the fluid-induced fracture propagation in porous media by fluid injection from the wellbore at a constant flow rate. The model is derived with the assumption that there is no gap between the front of the fluid and the fracture tip. Therefore, the KGD model essentially assumes the fracture propagation is in the fluid viscosity controlled regime, and the rock toughness is not explicitly considered $[28,45,60]$.

The analytical solution for the fracture half-length is given as

$L_{\mathrm{c}} / 2=C\left(\frac{q^{\prime 3} E}{\mu}\right)^{\frac{1}{6}} t^{\frac{2}{3}}$

where $q^{\prime}$ is the fracturing fluid injection rate per unit height of the crack, $E$ is the plane strain elastic modulus, and $\mu$ is the dynamic viscosity of fluid and $t$ is time. For the KGD model [30], $C=0.539$, and for the model proposed by Spence and Sharp [64], $C=0.515$.

The fracturing fluid is injected into an initial preexisting crack with a length of $2 \mathrm{~cm}$ in the numerical simulation, as shown in Fig. 13. The injection rate per unit height of the preexisting crack is assumed to be constant, $q^{\prime}=0.4 \times 10^{-5} \mathrm{~m}^{2} / \mathrm{s}$. The symmetry of the problem allows us to model only half of the space [12]. Figure 14 presents a comparison of fracture length between analytical solution and simulation. The result shows that the fracture length obtained from simulation agrees with that calculated by analytical solution. It is worth noting that KGD solution is given under viscosity-dominated regime, and hence, leak-off is not allowed (medium permeability $k=0$ ). In our simulation, the leak-off is allowed by considering permeable host medium. This may be the reason the fracture

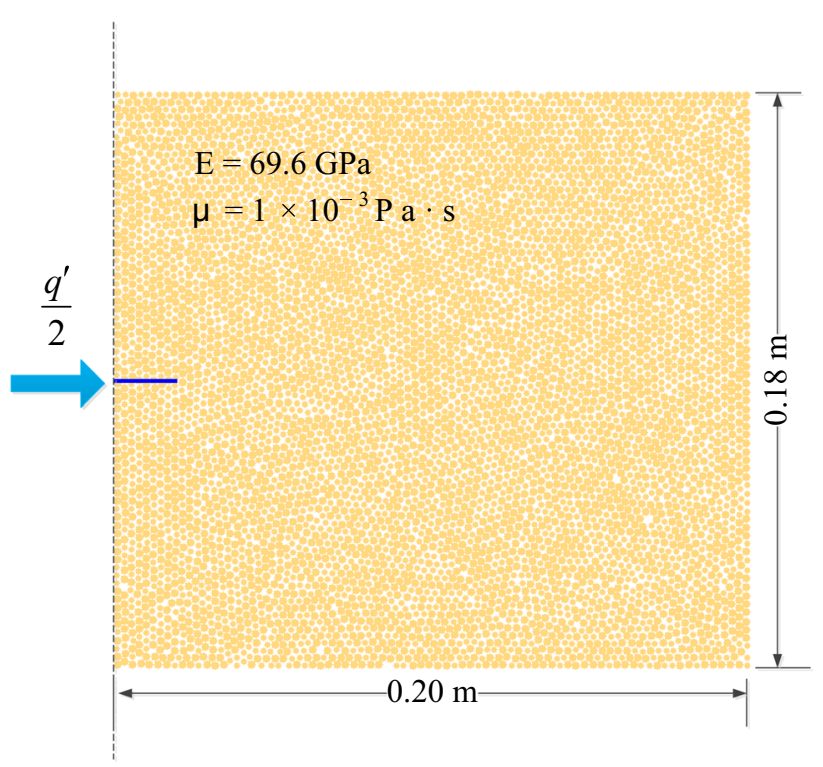

Fig. 13 Model used for hydraulic fracturing examples. The blue line indicates the initial flaw (color figure online) 


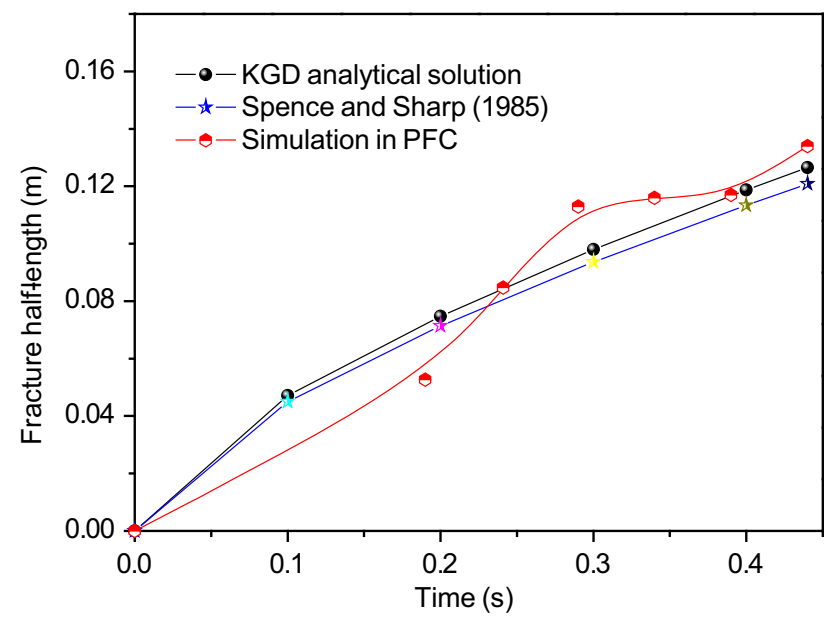

Fig. 14 Fracture half-length versus elapsed time from analytical solution and simulation. The cracks in PFC are micro-cracks caused by contact failure. The fracture length in this figure is obtained by measuring trajectory length of cracks. The solution given by Spence and Sharp [64] is calculated by Eq. (26) $(C=0.515)$

length in the simulation is lower than the result analytical solution in the beginning. Overall, the KGD model provides a good reference for the determination of hydraulic parameters. The fracture toughness of Lac du Bonnet granite is $K_{I C}=1.72 \mathrm{MPa} \cdot m^{1 / 2}$ [43]. The dimensionless toughness [1, 11] is about 2.74 in this research. The hydraulic fracture can be described the toughness-dominated regime. This implies the numerical solution with leak-off given by Bunger et al. [11] may be more suitable for the calibration of hydraulic parameters in this case.

\section{Hydraulic fracture in the specimens with two flaws}

The interaction and coalescence of cracks are important for understanding the mechanisms of hydraulic fracturing. In the following examples, we use a DEM network coupling model to simulate the interactions of cracks initiated at different locations and study the influence of flaws orientation on crack coalescence patterns. Here we consider a rectangular domain subjected to confining pressures of $1 \mathrm{MPa}$ in the $x$-direction and $1 \mathrm{MPa}$ in the $y$-direction. We introduce two flaws at different locations of the square domain and perform the DEM simulations with sufficient time step such that the DEM model reaches a new quasistatic equilibrium [18].

\subsection{Model setup}

The dimension of the numerical specimen is $20 \mathrm{~cm} \times 20$ $\mathrm{cm}$. A servo-control system is introduced in our numerical tests to keep the boundary stress constant. The servo-control system consists of four walls on the boundary of the specimen. The required stress state is achieved by moving the boundary walls. The assembly is initially created with confining stress of 0.1 MPa. Before the injection test, the particle assembly is loaded to specific stress state and the static equilibrium is maintained using the servo-control system. The numerical specimen is composed of particles of different sizes. The maximum radii of the particles are $144 \mathrm{~mm}$, and the minimum radii are $1.2 \mathrm{~mm}$. The radii of the particles are uniformly distributed. Two nearby flaws with different angles are configured in the modeled rock specimen (see Fig. 15), and the two preexisting flaws without bonding strength are placed into the model using

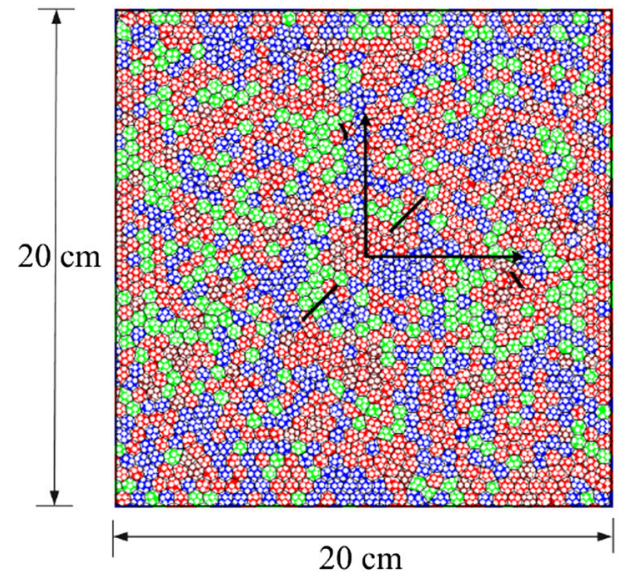

(a)

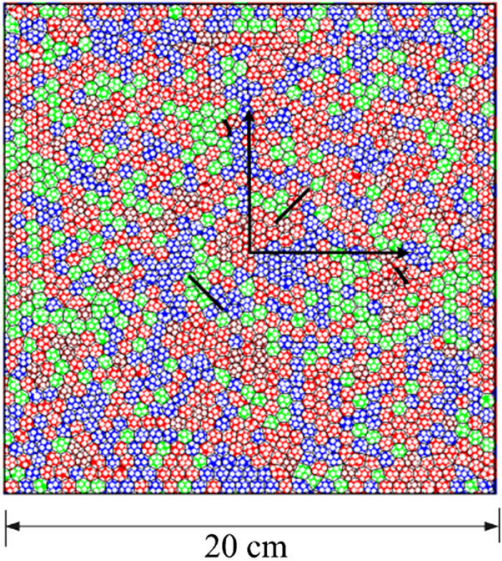

(b)

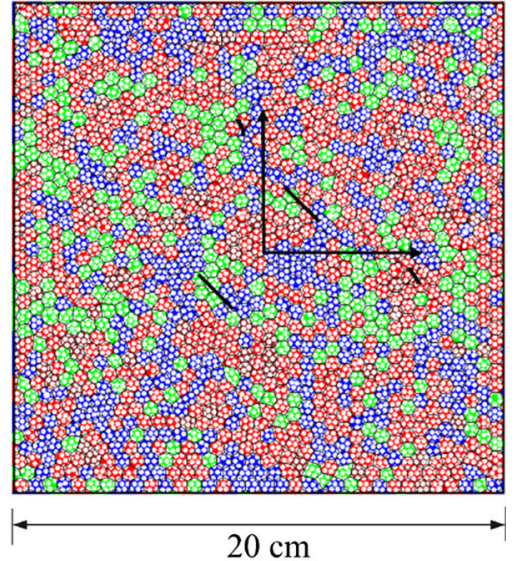

(c)

Fig. 15 Rock models with different nearby flaw patterns generated by the grain-based method. The red, green, blue, and brown areas indicate $\mathrm{k}$-feldspar, plagioclase, quartz, and biotite minerals, respectively. The mineral content is in accordance with the data given by Eberhardt et al. [25]. The black lines denote two preexisting flaws (color figure online) 

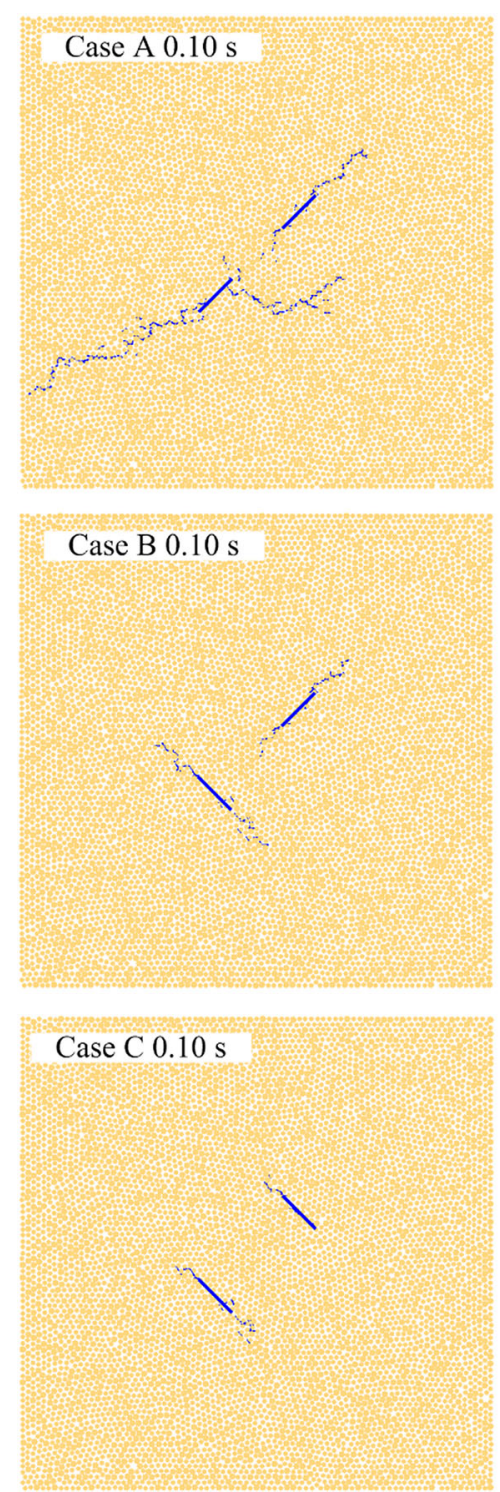
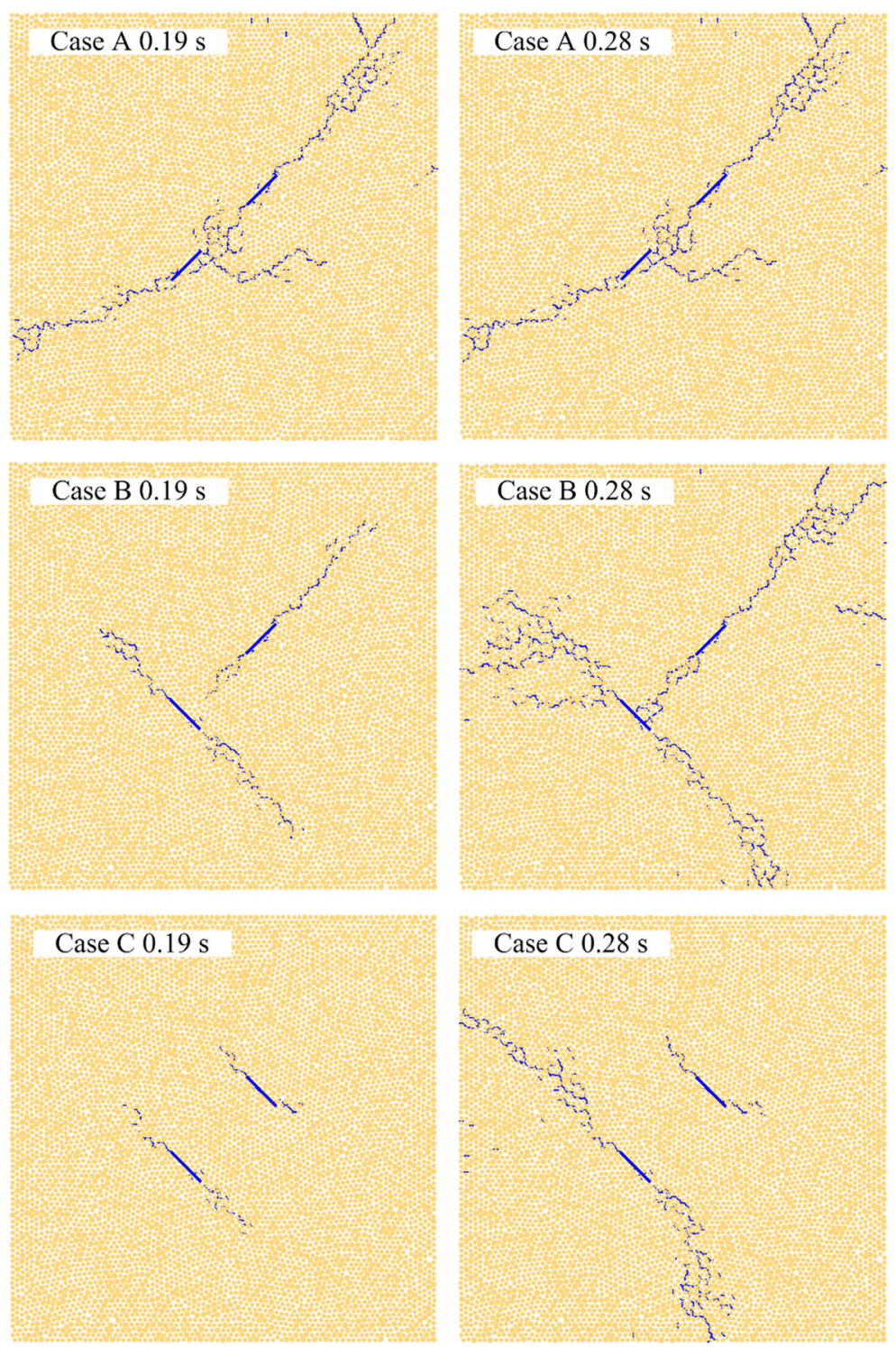

Fig. 16 Crack coalescence patterns with different inital flaw configurations. Each short line segment denotes a micro-crack

the smooth-joint model in PFC. The length of each initial flaws is $20 \mathrm{~mm}$, and the width is $2.4 \mathrm{~mm}$. The pressurized fluid is injected into the flaw area and diffuses with the cracks growth.

As shown in Fig. 15, case A consists of two preexisting flaws which are distributed symmetrically on the catercorner of the specimen to eliminate the effect of different stress states on the crack path [57]. Two flaws are perpendicular in case B, and they are placed in parallel in case C. The interval of the flaw center is $5 \mathrm{~cm}$. The pressurized fluid is injected into initial flaws with constant injection rate $2 \times 10^{-5} \mathrm{~m}^{2} / \mathrm{s}$. The crack propagation process initiated at the two flaws is monitored during the test. In this section, the effect of flaw configuration on the crack propagation is discussed. The confining stress of the sample is $\sigma_{x}=\sigma_{y}=1 \mathrm{MPa}$. Under this low confining stress, the rock specimens tend to fail by brittle, localized fracture [15]. The injection-induced cracks are expected to propagate and coalesce under different flaw configurations. The interaction mechanism for multiple fractures in brittle rock can be explained by the crack propagating trajectory.

\subsection{Crack propagation, interaction, and coalescence under different initial flaw patterns}

Figure 16 shows that the crack path is significantly influenced by the initial flaw orientation. For case A, the cracks lengthen and eventually meet to form a continuous crack along the line connecting the two flaws. It seems that two cracks in case A approach each other, and some small branches can be found at the fracture tip. Prior to the crack 
coalescence, the lower left fracture goes rightward and bypasses the frontier of another fracture. This implies the fractures avoid a tip to tip interaction, even though they may straightly move toward the other fracture. This phenomenon is also found in previous research [69]. In case B, the two leading cracks turn to intersect at about 90 degrees. In the beginning, two leading fractures grow and extend along the direction of preexisting flaws. The fracture tips show some branches after two fractures get to coalesce. When two initial flaws are parallel, the two leading cracks begin to propagate in a parallel direction at the beginning and tend to deviate from each other later. The growth rate of crack initiation from upper right flaw is slower than that from the lower left flaw in case $\mathrm{C}$. The reason is that the microstructure in the specimen represented by the grainbased model is not strictly homogeneous. Therefore, the areas around two preexisting flaws may have different microstructure and strength characteristic due to the spatial heterogeneity.

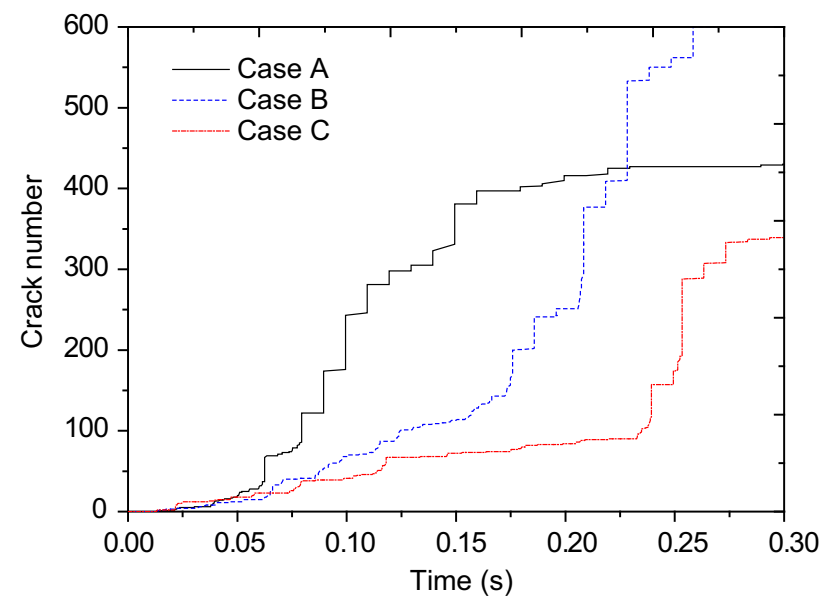

Fig. 17 Crack number for different flaw configurations
The brittle behavior is highly significant for granite especially if the confining pressure is low. Consequently, cracks may grow very fast. Remarkably, according to the time history exhibited in each figure, the rate of crack growth is the highest when the two initial flaws are aligned. In this case, the cracks start to coalesce at about $0.10 \mathrm{~s}$ for case A. However, at the same time instant of cases B and $\mathrm{C}$, the cracks merely began to growth near the flaws. Figure 17 shows the number of cracks in the three cases. It is obvious that the distribution and orientations of initial flaws affect the crack trajectory, crack propagation rate and crack number. Case A shows the fastest growing rate of crack (see Fig. 17). This indicates that there is a strong acceleration effect when the two flaws are aligned with each other and this configuration promotes the generation of new cracks.

The crack propagation rate in the specimen can be explained by Eq. (27). According to the subcritical crack (stress corrosion) propagation law [6], the crack propagation rate, $v$, can be obtained by the mode I stress intensity factor, $K_{I}$, the subcritical index of the material, $n$ and an empirically derived constant, $c$. Equation (27) indicates that even minor crack interaction, which will result in the variation of $K_{I}$, can finally lead to an exponential increase in crack propagation rates. This explains why the crack number curve shows a tremendous jump in the stage of crack interaction. It suggests that an appropriate design of injection hole with strong positive interaction will tremendously enhance the efficiency of hydrocarbon recovery.

$v=c K_{I}^{n}$

As shown in Fig. 18, the crack grows along the path perpendicular to the black compressive force chain. It is interesting to note that this result seems to agree with the fluid grain displacement regimes from fluid injection into a

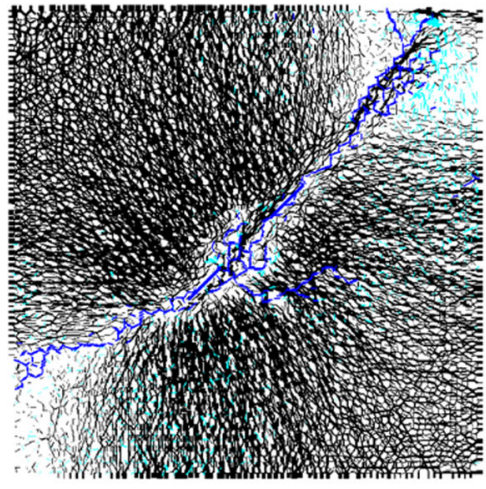

Case A $0.28 \mathrm{~s}$

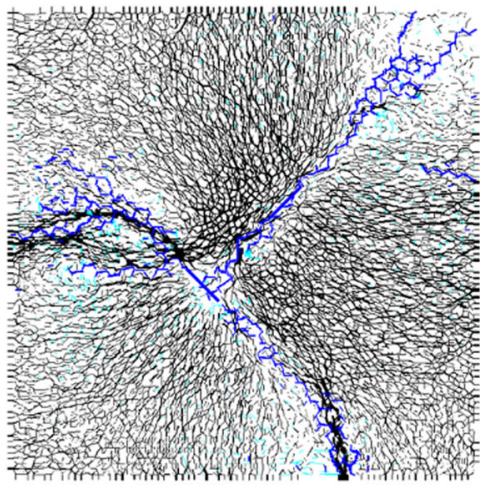

Case B $0.28 \mathrm{~s}$

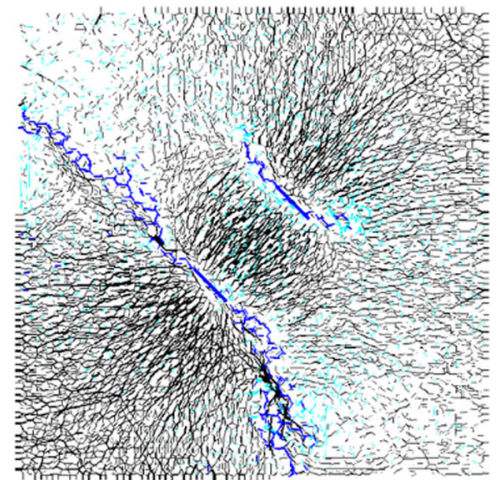

Case C $0.28 \mathrm{~s}$

Fig. 18 Distribution of contact force chain for three cases. The black lines indicate the compressive force chain, the cyan lines indicate the tensile force chain, the blue lines represent cracks caused by the tensile failure, and the bold line segments denote two initial flaws (color figure online) 
dense granular media [80]. The tensile force converges on the tip of propagated cracks. This suggests that the failure and slippage of contacts are most likely to arise on this intensive tensile zone.

In previous studies, the area ahead of the crack tip is termed as a process zone [75] where small-scale yielding, micro-cracking or void growth and coalescence may take place continually. The size of the process zone can be estimated by the tensile area in the force chain. Once the micro-cracks in the process zone link together, the macrocracks may extend beyond the process zone. When the two flaws align and close to each other, their process zones are likely to overlap. This may contribute to the fast propagation of cracks due to development of dense micro-cracks and the strength degradation in this overlapped process zone. This supports the notion that fracture propagation is likely affected by the stress fields of adjacent fluid-driven fracture.

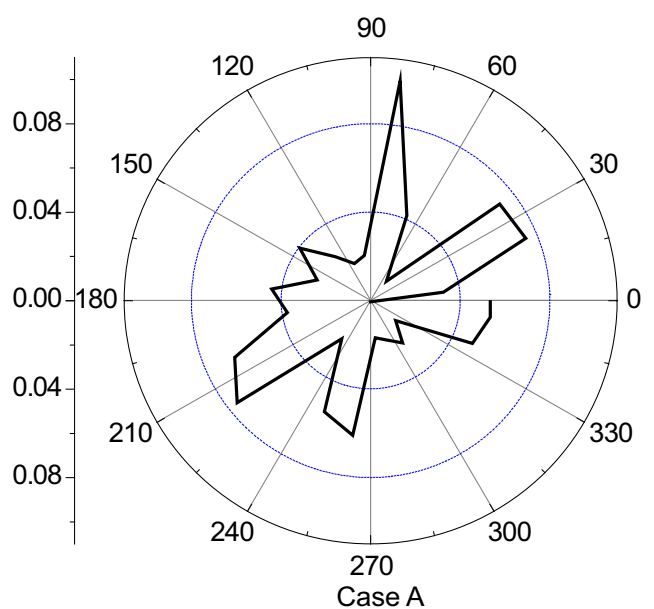

(a)

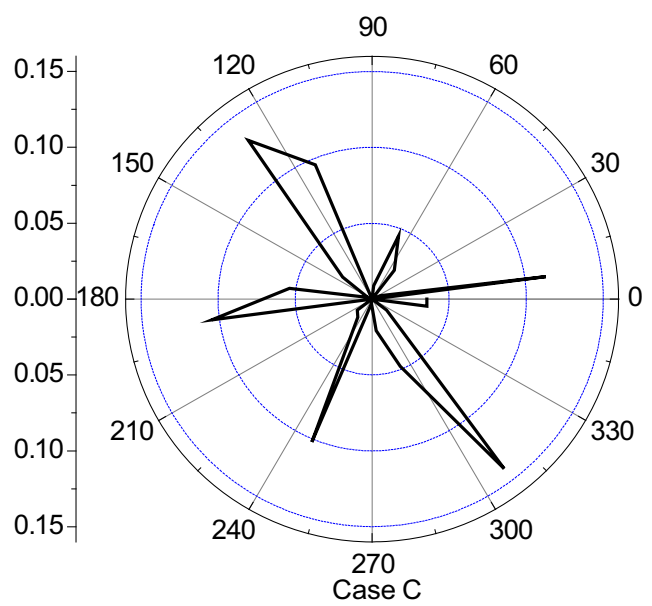

(c)
A statistical analysis for crack orientation at the time of $0.28 \mathrm{~s}$ is given to quantitatively evaluate the effect of preexisting flaws on crack interaction and coalescence (Fig. 19). The bond breaks when either the tensile normal or shear contact force exceeds the corresponding bond strength. The failure of each contact generates a new crack passing through the contact point between two particles. The orientation of each crack is defined by a unit direction vector perpendicular to the line of two particles. The angles of cracks are calculated by the unit direction vector of cracks.

The cracks are counted in each azimuthal bins of $15^{\circ}$, from $0^{\circ}$ to $360^{\circ}$. The result reveals that the crack paths largely depend upon the orientation of initial flaws in the numerical specimen. As shown in Fig. 19a, the orientation of most cracks falls in the area about $15^{\circ}-90^{\circ}$ and $195^{\circ}$ $270^{\circ}$ when two flaws are aligned. Cases $\mathrm{B}$ and $\mathrm{C}$ have a concentrated crack orientation. Especially for case C, cracks are concentrated on the orientation of $120^{\circ}$ and

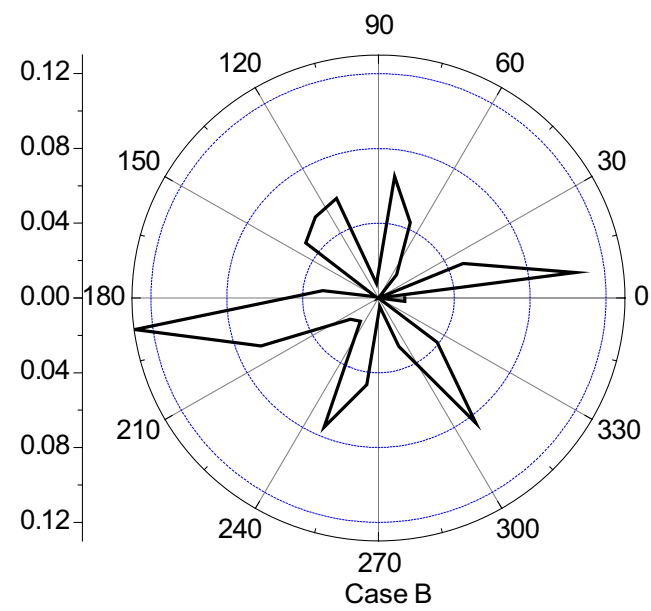

(b)

Fig. 19 Crack orientations for different configuration of preexisting flaws. Crack orientations above is obtained at the time of $0.28 \mathrm{~s}$ 


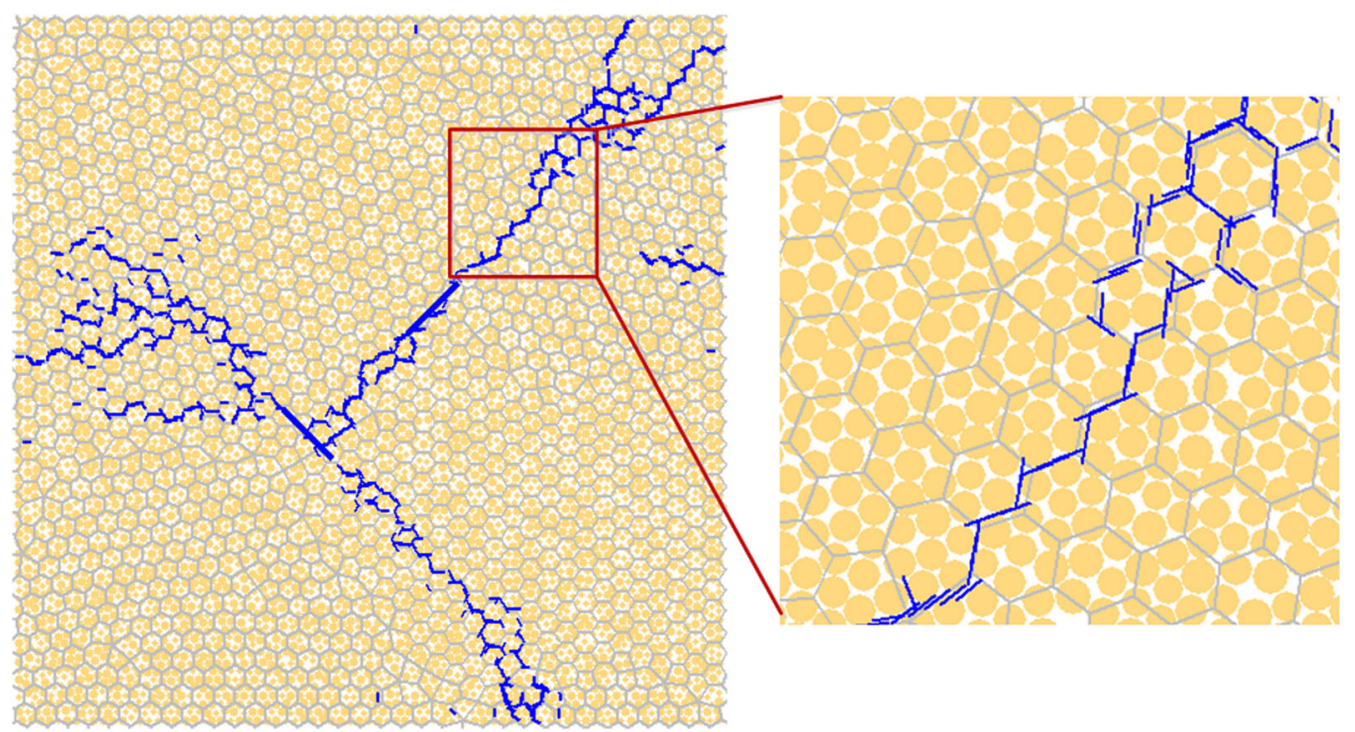

Fig. 20 Propagation of cracks along the grain boundary. The blue lines are cracks, and the gray polygons are grains (color figure online)

$310^{\circ}$. The far-field principal stress for different flaw orientation is the same, but the orientation of crack is distinctly different. The crack orientation indicates that local stress field determined by initial flaw direction dominates the propagation of cracks.

At the grain level, micro-cracks tend to propagate along the grain boundary (Fig. 20) because the intra-grain strength is much higher than the inter-grain strength. This is the reason that orientations of crack in Fig. 19 are not in strict accordance with the macroscopic direction of crack in Fig. 14.

Figure 21 shows the water pressure distribution obtained from simulations with different initial flaw configurations. It is obvious that water pressure mainly distributes on the crack area. The crack path becomes the major fluid channel in the specimens due to the increase in aperture at the failure contacts. The pore pressure mainly distributes along the crack path, because the micro-cracks alter the permeability greatly and facilitate the pressure dissipation. The macroscopic cracks seem continuous; however, some micro-cracks are not connected in the microscopic numerical model. This discrepancy may be attributed to the over-simplistic formation mechanism of cracks in the model as well as the deficiencies brought by the 2D fluid pore network model. These factors may lead to the discontinuity of water pressure in some regions. The water pressure is higher in the cases where the two initial flaws are aligned with each other. This high pore water pressure may explain that the growth rate of crack in case A is relatively faster compared with other two cases.

Once we obtain the local permeability of each fluid channel, the shortest path algorithm can be used to find the effective fluid flow path between two given points. Here, local permeability indicates the permeability for each fluid channel, which is related in Fig. 4. Darcy's law can be written as

$q=\frac{k A \Delta P}{\mu L}$

where $k$ is the permeability, in $\mathrm{m}^{2}$ and $\mathrm{A}$ is the fluid area. To obtain the local permeability in each fluid channel, we assume that the fluid area is equal the aperture of pore in the 2D model. By combining Eqs. (5) and (28), the local permeability for each fluid channel, $k$, in $\mathrm{m}^{2}$, can be expressed as $q=\frac{a^{2}}{12}$. So the local permeability for each fluid channel can be calculated by the aperture of pore. The fluid channel in the model without crack maintains the initial local permeability $2.08 \times 10^{-14} \mathrm{~m}^{2}$, which is far lower than the permeability of fluid channel in the crack area, which is on the order of $10^{-9} \mathrm{~m}^{2}$.

\subsection{Analysis of fluid and crack networks}

After calculating the permeability of each fluid channel, a weighted graph is generated to represent the flow network. This weighted graph [42] is a network connecting the pore centers of each void space (see Fig. 4) connected by each flow channel, and the flow channel has a weight of $l_{i} / k_{i} . l_{i}$ is the distance of two pore centers connected by channel $i$, and $k_{i}$ is the permeability of channel $i$. The value of $l_{i} / k_{i}$ is selected as the weight for the fluid channel in the network, as this value can reflect the transmission capacity of the fluid channel [see Eq. (28)]. One of the most important properties to study the properties of a network is the shortest path between two vertices. Dijkstra's algorithm 

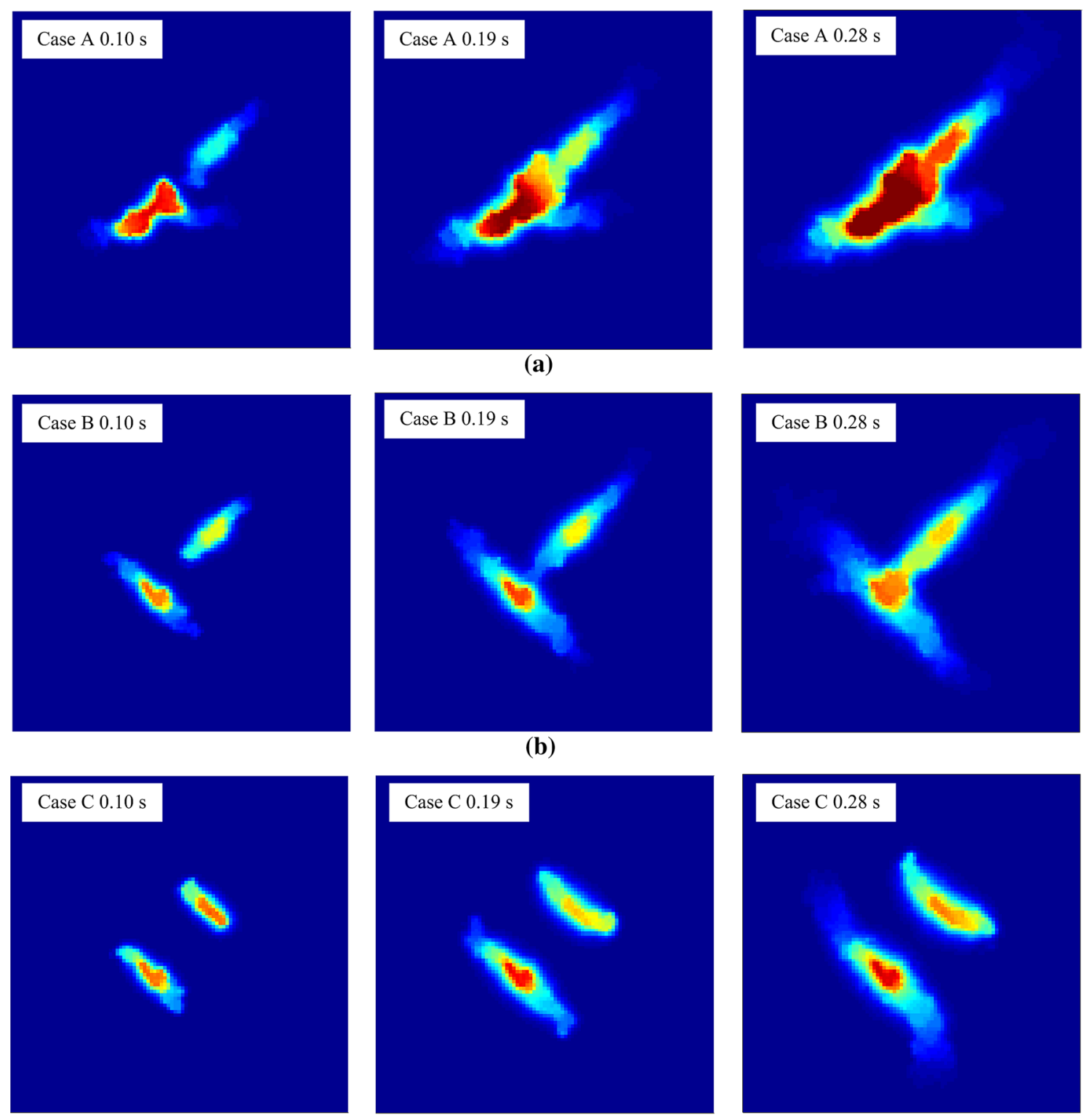

(b)

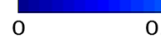

0.5

(c)

Fig. 21 Water pressure distribution for different flaw configurations. This figure is plotted by MATLAB using the pressure data of pore centers

[23] is used to seek the shortest path between two injection points.

In the flow network weighed graph, pore centers are represented by vertices and flow channels by edges. Dijkstra's algorithm works as follows [67]: (1) Identify one of the vertices in one injection point as the first active vertex, and one of the vertices on the other injection point as targeted outflow vertex. (2) Consider all of the unvisited vertices around active vertex and calculate their tentative distances by the weights. Compare the newly calculated tentative distance with the currently assigned value and assign the smaller one. After that, remove the active vertex from the unvisited set. (3) This algorithm stops once the targeted outflow vertex becomes an active vertex. Otherwise, select the unvisited node that is marked with the smallest tentative distance, set it as the new active vertex and repeat step (2).

Figure 22 presents the shortest path between two centers of flaws using Dijkstra's algorithm. This analysis predicts the flow path only by local permeability, and it coincides exactly with the crack path. When the two flaws are on a line, the flow path goes straightly from one injection point to the other, only through 36 vertices on the weight graph. While the flow paths for cases of orthometric flaw and 


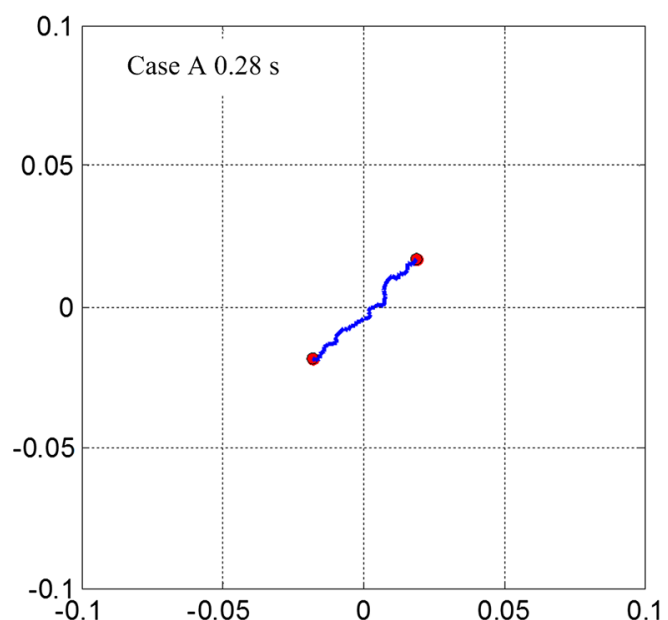

(a)

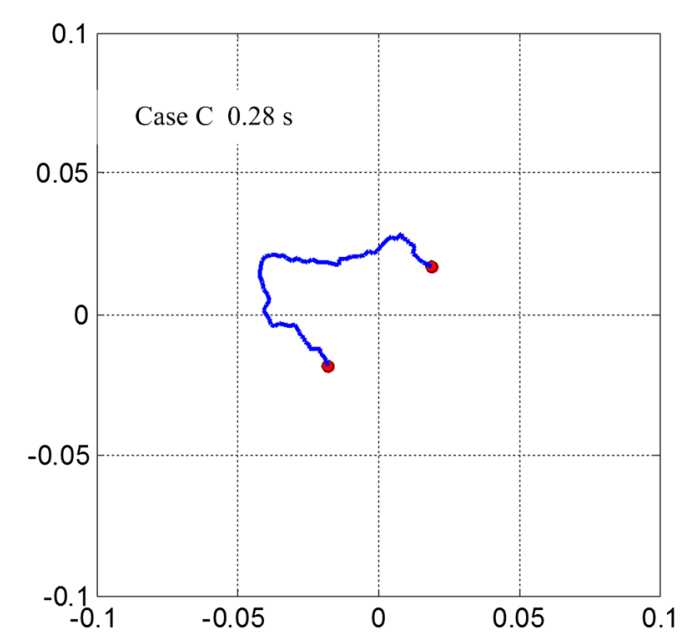

(c)

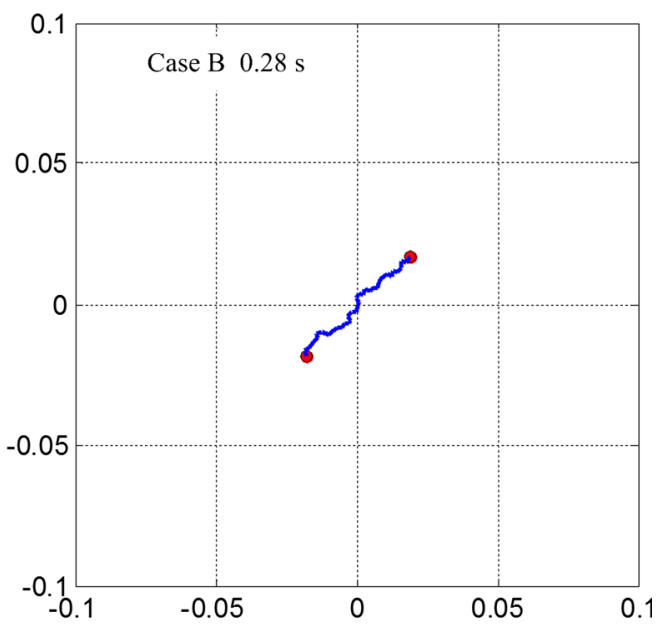

(b)

(1)

Fig. 22 The shortest flow path between two injection points by Dijkstra's algorithm. The weight for each edge is $\frac{l_{i}}{k_{i}}$

paralleled flaw go through 36 and 67 vertices, respectively. The geometrical tortuosity, $\tau=L_{\mathrm{e}} / L_{\mathrm{s}}$, is often used to quantify the complexity of the flow network, which is defined here as the ratio between the effective length of the shortest flow path, $L_{\mathrm{e}}$, and the injection point spacing, $L_{\mathrm{s}}$ [67]. The widely used Kozeny-Carman equation estimates that the effective permeability is proportional to the reciprocal of tortuosity $1 / \tau$ [67]. From Fig. 22 , it can be seen that the geometrical tortuosity for case $\mathrm{C}$ is conspicuously higher. Thus, the effective permeability for the shortest flow path in case $\mathrm{C}$ is smaller than that in other two cases. However, this effective permeability is an evaluation only for the shortest flow path between two specific points. The fluid transportation capacity in the whole crack network will be discussed in detail below.

In this research, a new crack is formed due to the failure of a contact. In order to study the topological property of the crack network, we introduce some efficiency measures in graph theory to see how well the fluid diffuses over the crack graph. The blue lines represent cracks, and the red dots indicate the pore centers (see Fig. 23). The crack networks are extracted from the final stage in Fig. 16. This undirected graph can be considered as a network with $N$ vertices (nodes) and $K$ edges (links or connections). Here, the graph $\boldsymbol{G}$ can be a weighted and possibly even non-connected and non-sparse graph [38]. This graph $\boldsymbol{G}$ is described by two matrixes, the adjacency matrix, $\left\{a_{i j}\right\}$ and the matrix of the weights associated with each link, $\left\{l_{i j}^{\prime}\right\}$. $a_{i j}$ is defined as a set of numbers, $a_{i j}=1$ when there is a connection or edge joining vertex $i$ to $j$, and $a_{i j}=0$ otherwise. $l_{i j}^{\prime}$ is identified with the weight of edge joining vertex $i$ to $j$. In such a situation, let the weight of edge joining vertices $i$ to $j, l_{i j}^{\prime}$ equal the length of the edge, when there is an edge joining vertex $i$ to $j$. The definition about the weight of edge was also adopted by Valentini et al. [70] to analyze rock fracture network. We assume that the 


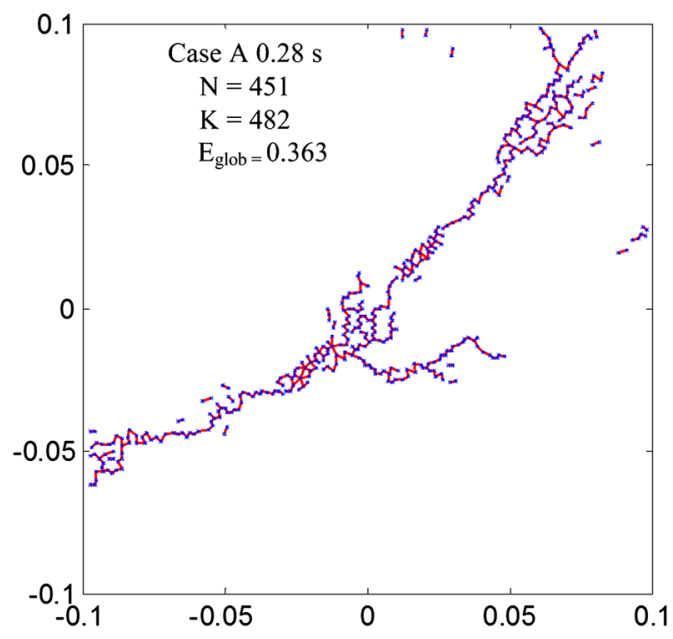

(a)

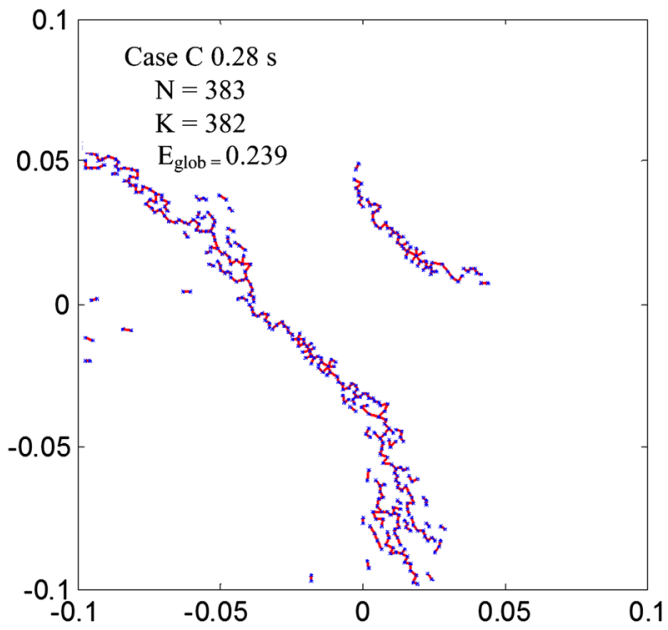

(c)

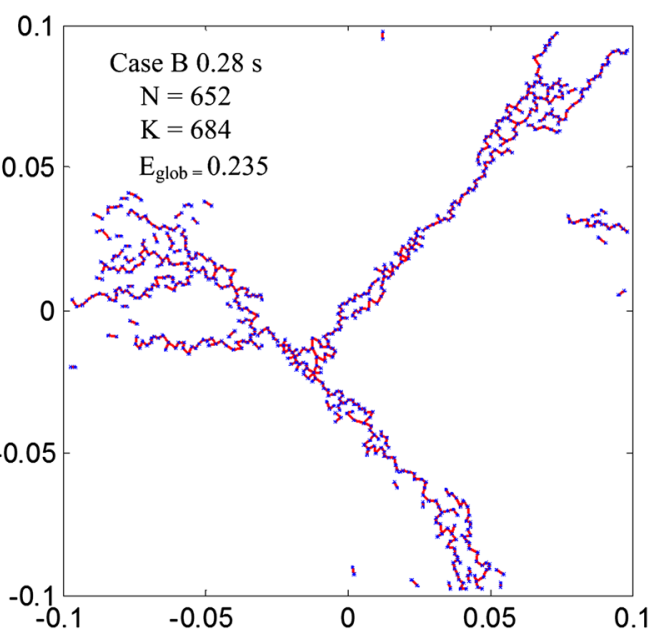

(b)

Fig. 23 Crack graph at time of $0.28 \mathrm{~s}$. The red lines are cracks, and the blue dots are pore centers in the network (color figure online)

weight of an edge is zero, $l_{i j}^{\prime}=0$, when there is no edge between $i$ and $j$. We also define $l_{i j}^{\prime}=0 \forall i=j$.

The shortest path between vertices $i$ and $j$ is denoted by $d_{i j}$. The shortest path $d_{i j}$ can be obtained by Dijkstra's shortest path algorithm. Actually, $d_{i j}$ between two generic points $i$ and $j$ is the minimum sum of weight $l_{i j}^{\prime}$ throughout all the possible paths in the graph from $i$ to $j$. Since the pressurized hydraulic fluid mainly transfers along the crack path, it is reasonable to adopt the shortest path, $d_{i j}$, to evaluate efficiency of the network. Once we get the shortest path, $d_{i j}$, we can simply estimate the efficiency, $\zeta_{i j}$ in the communication between vertices $i$ to $j$ by the reciprocal of the shortest distance:

$\zeta_{i j}=\frac{1}{d_{i j}} \forall i, j$

Consequently, the global efficiency $E(\boldsymbol{G})$ of the whole graph $\boldsymbol{G}$ can be defined as [70,71]
$E(\mathbf{G})=\frac{\sum_{i \neq j \in \mathbf{G}} \zeta_{i j}}{N(N-1)}=\frac{1}{N(N-1)} \sum_{i \neq j \in \mathbf{G}} \frac{1}{d_{i j}}$

If there is no link between vertices $i$ and $j$, we assume $d_{i j}=+\propto$ and then get $\zeta_{i j}=0$. Equation (30) gives the efficiency $E(\boldsymbol{G})$ which varies in the range $[0,+\propto] . E(\boldsymbol{G})$ is normalized to be in the range of $[0,1]$, by introducing an ideal graph $\mathbf{G}^{\mathbf{i}}$. In the ideal graph $\mathbf{G}^{\mathbf{i}}$, every two vertices generate an edge, in total $N(N-1) / 2$ edges. So we can obtain the maximum value of efficiency on this ideal graph with $d_{i j}=l_{i j}^{\prime} \forall i=j$. The maximum efficiency is given by Latora and Marchiori [38].

$E\left(\mathbf{G}^{\mathbf{i}}\right)=\frac{1}{N(N-1)} \sum_{i \neq j \in \mathbf{G}^{\mathbf{i}}} \frac{1}{l_{i j}^{\prime}}$

The normalized global efficiency $E_{\text {glob}}$, therefore, is written as 


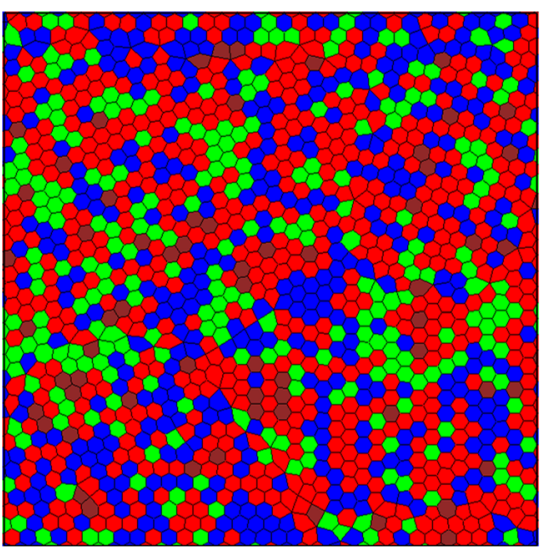

Specimen 0

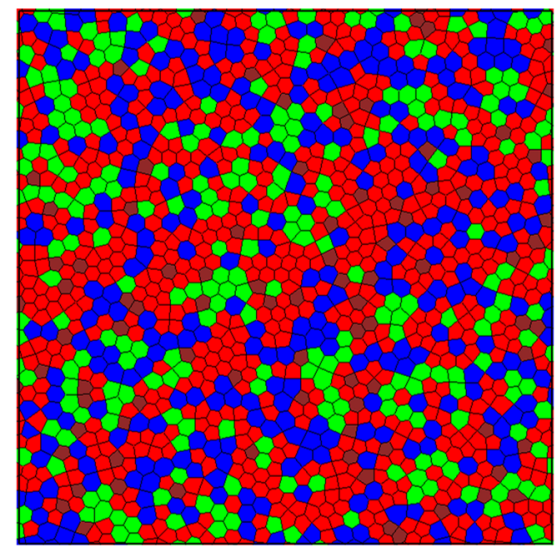

Specimen 1

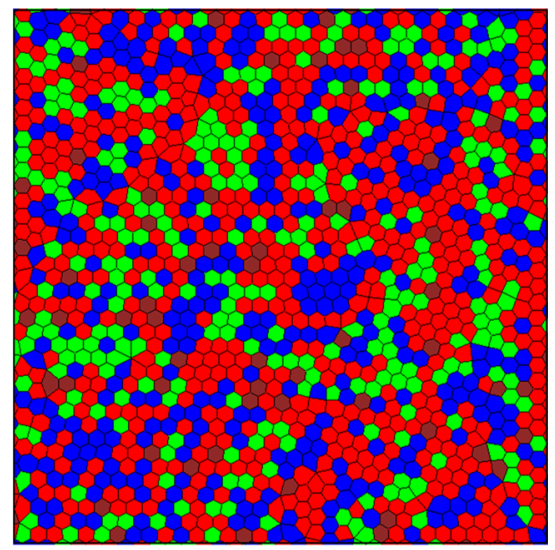

Specimen 2

Fig. 24 Three specimens with different grain structures. The red, green, blue, and brown areas indicate k-feldspar, plagioclase, quartz, and biotite, respectively. Specimen 0 is used in the simulation in Sects. 3 and 4 (color figure online)

$E_{\text {glob }}=\frac{E(\mathbf{G})}{E\left(\mathbf{G}^{\mathbf{i}}\right)}$

The global efficiency $E_{\text {glob }}$ of the crack network is calculated by using above method. For the crack graph of cases $\mathrm{A}, \mathrm{B}$, and $\mathrm{C}$, the corresponding global efficiency is $0.363,0.235$, and 0.239 , respectively. Figure 23 shows that two preexisting flaws in the same direction may lead to a well-connected crack network with high global efficiency. The crack graph shows the relatively high global efficiency, which has the same topology as in the natural fracture and small-world network $\left(E_{\text {glob }}>0.1\right)$ [70, 71]. Based on the illustration above, the large global efficiency means good connectivity in crack network. The crack networks in cases $\mathrm{B}$ and $\mathrm{C}$ have similar global efficiency. It is reasonable that crack network in case $\mathrm{C}$ has a low global efficiency because its two fractures do not coalesce. The crack network in case B has a lot of discontinuous branches at the crack tip, which results in a low global efficiency. The global efficiency of crack network in case A is the highest among three cases. This reveals that materials with micro-cracks are well connected are more likely to form a major fracture when two initial flaws are parallel.

\section{Discussion}

In this research, the degradation of stiffness, Young's modulus, and Poisson's ratio caused by the progressive accumulation of damage in the sample is negligible and the contact model is a linear elastic model. However, the coalescence of interacting cracks may be accompanied by accumulation of damage as the bridging zone between the cracks weakens and collapses [26]. In addition, the strength reduction induced by water pressure is also neglected. If the degradation of strength induced by accumulation of damage and water is considered, the breakdown pressure would be smaller and the growth rate of crack would be faster.

It should be noted that the spatial heterogeneity caused by initialization algorithm, grain positions, grain size, and bond strength also influences the propagation of crack $[41,44]$. We mainly focus on the effect of initial flaws on the crack pattern in this research. Although our grain structure is not from a slice scan images of real rock, the numerical model of GBM has been proved to be feasible to simulate the macro-mechanical behavior of rock $[7-9,52]$. To completely reproduce material microstructure, it is necessary to extract the grain structure from material micro-tomography images $[4,5]$. This will be considered in our further work.

To evaluate the effect of heterogeneity caused by initialization algorithm, grain positions, grain size, and bond strength, we generate two additional specimens (specimens 1 and 2) of different grain structures (see Fig. 24). The mean grain size for three specimens is nevertheless identical. Then, we repeat the simulations of the three crack orientation scenarios which are labeled as cases A1, B1, C1, A2, B2, and C2 (Fig. 25). As shown in Fig. 25, the crack pattern is also influenced by the grain structure. The crack patterns from two specimens indicate that the simulated cracks mainly propagate along the direction of original flaw. The scenario with two aligned initial flaws still shows the fastest propagation rate of crack. This result is in accordance with the previous conclusion.

\section{Conclusions}

The propagation, interaction, and coalescence of cracks are of vital importance for numerous geoengineering applications, such as unconventional hydrocarbon recovery, 

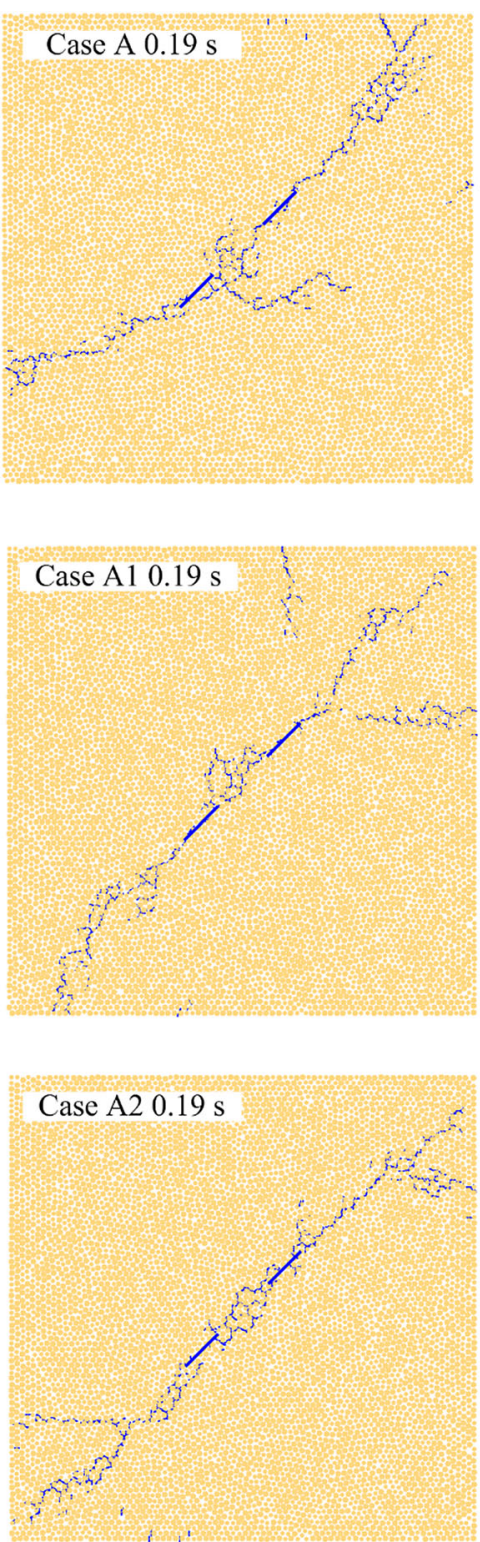

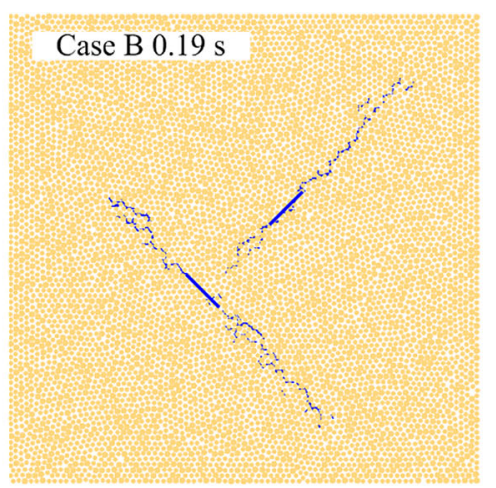

(a) Specimen 0

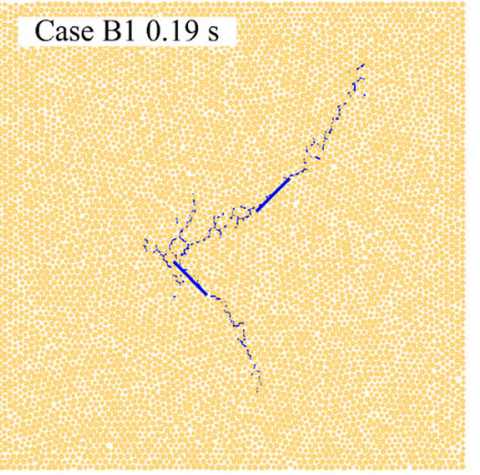

(b) Specimen 1

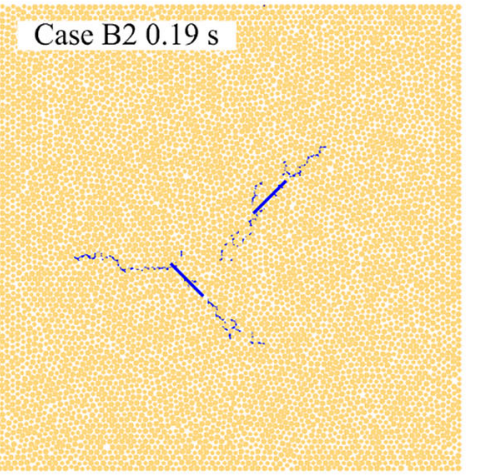

(c) Specimen 2

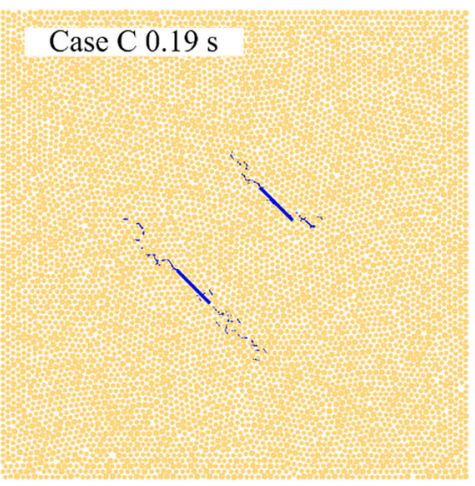

Case $\mathrm{C} 10.19 \mathrm{~s}$

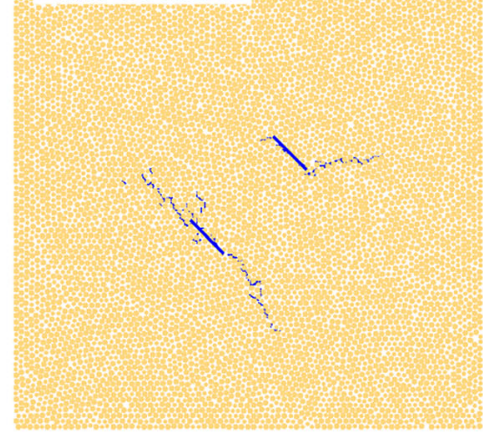

Case C2 $0.19 \mathrm{~s}$

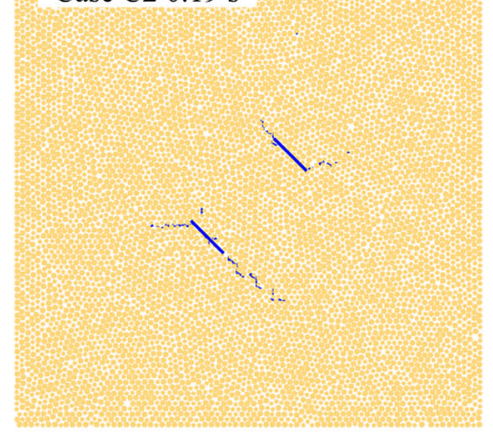

Fig. 25 Crack patterns considering different grain structure

geological storage of carbon dioxide, and nuclear waste. This research focuses on analyzing the propagation, interactions, and coalescence of two nearby fluid-driven fractures with a coupled flow-network/discrete element model. A new scheme to improve the computational efficiency is proposed, and the variation of pore network induced by fracture propagation is considered in the improved DEM network coupling model. The material parameters are calibrated based on the macro-properties of Lac du Bonnet granite and KGD solution. By using the grain-based model, the macro-mechanical properties, especially the ratio of the compressive strength to tensile strength, can be captured.
Using the improved DEM network coupling model, we conduct a series of numerical simulations in which two initial flaws are placed with different patterns in synthetic rock. The influence of flaw orientation on the coalescence of fluid-driven fracture is studied by numerical tests. Results indicate that cracks show higher propagation rate when two flaws distribute in a straight line. Depending on the flaw direction, fluid-driven cracks originated from two different flaws may join together in the same propagation direction, or they may meet at different angles. The coalescence patterns originated from the two flaws are closely associated with flaw direction. When the two flaws are aligned with each other, the macroscopic cracks coalesce in 
a straight line. These interaction mechanisms are analyzed via geometrical measures from graph theory, in which the crack network enhanced by the fluid-induced fractures is analyzed systematically. The results reveal that multiple initial flaws in the same direction may lead to a wellconnected crack network with high global efficiency.

Acknowledgements This research is supported by the Earth Materials and Processes program at the US Army Research Office under grant contracts W911NF-14-1-0658 and W911NF-15-1-0581, Air Force Office of Scientific Research under grant contract FA9550-1186-171-0169, US Department of Energy Nuclear Engineering University Program under grant contract DE-NE0008534, National Science Foundation under grant contract EAR-1516300, Anhui Science and technology research projects (No. 1604a0802106), the Open Fund of the Key Laboratory of Rock Mechanics in Hydraulic Structural Engineering, Ministry of Education, Wuhan University, the Open fund from state Key Laboratory of Water Resources and Hydropower Engineering Science, China (No. 2016SGG02), the Open Fund of the Key Laboratory of Rock Mechanics in Hydraulic Structural Engineering, Ministry of Education, Wuhan University, Key Laboratory of Geological Hazards on Three Gorges Reservoir Area (China Three Gorges University), Ministry of Education (No. 2015KDZ03), and Chinese Universities Scientific Fund (No. JZ2016HGBZ1021). These supports are gratefully acknowledged. The first author is also grateful to the China Scholarship Council (CSC) for providing him with a scholarship during his study in the USA. These supports are gratefully acknowledged.

\section{References}

1. Adachi JI, Detournay E (2008) Plane strain propagation of a hydraulic fracture in a permeable rock. Eng Fract Mech 75(16):4666-4694

2. Adachi J, Siebrits E, Peirce A, Desroches J (2007) Computer simulation of hydraulic fractures. Int J Rock Mech Min Sci 44(5):739-757

3. Al-Busaidi A (2005) Distinct element modeling of hydraulically fractured Lac du Bonnet granite. J Geophys Res 110:B06302. https://doi.org/10.1029/2004jb003297

4. Al-Raoush R, Alshibli KA (2006) Distribution of local void ratio in porous media systems from 3D X-ray microtomography images. Physica A 361(2):441-456

5. Al-Raoush R, Willson C (2005) Extraction of physically realistic pore network properties from three-dimensional synchrotron $\mathrm{X}$-ray microtomography images of unconsolidated porous media systems. J Hydrol 300(1):44-64

6. Atkinson BK (1984) Subcritical crack growth in geological materials. J Geophys Res Solid Earth (1978-2012) 89(B6):4077-4114

7. Bahrani N, Kaiser PK, Valley B (2014) Distinct element method simulation of an analogue for a highly interlocked, non-persistently jointed rockmass. Int J Rock Mech Min Sci 71:117-130

8. Bewick RP, Kaiser PK, Bawden WF, Bahrani N (2013) DEM, simulation of direct shear: 1. Rupture under constant normal stress boundary conditions. Rock Mech Rock Eng 47(5):1647-1671

9. Bewick RP, Kaiser PK, Bawden WF (2013) DEM, simulation of direct shear: 2. Grain boundary and mineral grain strength component influence on shear rupture. Rock Mech Rock Eng 47(5):1673-1692
10. Boone TJ, Ingraffea AR, Roegiers J-C (1991) Simulation of hydraulic fracture propagation in poroelastic rock with application to stress measurement techniques. Int J Rock Mech Min Sci Geomech Abstr 28:1-14

11. Bunger AP, Detournay E, Garagash DI (2005) Toughness-dominated Hydraulic Fracture with Leak-off. Int J Fract 134(2):175-190

12. Carrier B, Granet S (2012) Numerical modeling of hydraulic fracture problem in permeable medium using cohesive zone model. Eng Fract Mech 79:312-328

13. Cho N, Martin CD, Sego DC (2007) A clumped particle model for rock. Int J Rock Mech Min Sci 44(7):997-1010

14. Choo J, Sun W (2018) Cracking and damage from crystallization in pores: Coupled chemo-hydro-mechanics and phase-field modeling. Comput Methods Appl Mech Eng 335:347-379

15. Choo J, Sun W (2018) Coupled phase-field and plasticity modeling of geological materials: From brittle fracture to ductile flow. Comput Methods Appl Mech Eng 330:1-32

16. Clark JB (1949) A hydraulic process for increasing the productivity of wells. J Petrol Technol 1(01):1-8

17. Cook NGW (1992) Natural joints in rock: mechanical, hydraulic and seismic behaviour and properties under normal stress. Int $\mathrm{J}$ Rock Mech Min Sci Geomech Abstr 29:198-223

18. Cook BK, Lee MY, DiGiovanni AA, Bronowski DR, Perkins ED, Williams JR (2004) Discrete element modeling applied to laboratory simulation of near-wellbore mechanics. Int $\mathrm{J}$ Geomech 4(1):19-27

19. Cui ZD, Liu DA, Zeng RS, Niu JR, Wang HJ, Shi XS (2013) Resistance of caprock to hydraulic fracturing due to $\mathrm{CO}_{2}$ injection into sand lens reservoirs. Eng Geol 164:146-154

20. Dahi Taleghani A (2009) Analysis of hydraulic fracture propagation in fractured reservoirs: an improved model for the interaction between induced and natural fractures. The University of Texas at Austin, Austin

21. Detournay E (2004) Propagation regimes of fluid-driven fractures in impermeable rocks. Int J Geomech 4(1):35-45

22. Detournay E (2016) Mechanics of hydraulic fractures. Annu Rev Fluid Mech 48:311-339

23. Dijkstra EW (1959) A note on two problems in connexion with graphs. Numer Math 1(1):269-271

24. Downie R, Kronenberger E, Maxwell SC (2010) Using microseismic source parameters to evaluate the influence of faults on fracture treatments: a geophysical approach to interpretation. In: SPE annual technical conference and exhibition: society of petroleum engineers

25. Eberhardt E, Stimpson B, Stead D (1999) Effects of grain size on the initiation and propagation thresholds of stress-induced brittle fractures. Rock Mech Rock Eng 32(2):81-99

26. Eberhardt E, Stead D, Stimpson B (1999) Quantifying progressive pre-peak brittle fracture damage in rock during uniaxial compression. Int J Rock Mech Min Sci 32(2):361-380

27. Falls SD, Young RP, Carlson SR, Chow T (1992) Ultrasonic tomography and acoustic emission in hydraulically fractured Lac du Bonnet grey granite. J Geophys Res Solid Earth (1978-2012) 97(B5):6867-6884

28. Fu P, Johnson SM, Carrigan CR (2013) An explicitly coupled hydro-geomechanical model for simulating hydraulic fracturing in arbitrary discrete fracture networks. Int J Numer Anal Meth Geomech 37(14):2278-2300

29. Gale JFW, Reed RM, Holder J (2007) Natural fractures in the Barnett Shale and their importance for hydraulic fracture treatments. AAPG Bull 91(4):603-622

30. Geertsma J, De Klerk F (1969) A rapid method of predicting width and extent of hydraulically induced fractures. J Petrol Technol 21(12):571-581 
31. Helmons RLJ, Miedema SA, Alvarez Grima M, van Rhee C (2016) Modeling fluid pressure effects when cutting saturated rock. Eng Geol 211(Supplement C):50-60

32. Hunsweck MJ, Shen Y (2013) Lew Aa, n J. A finite element approach to the simulation of hydraulic fractures with lag. Int $\mathbf{J}$ Numer Anal Meth Geomech 37(9):993-1015

33. Ishida T, Chen Q, Mizuta Y, Roegiers JC (2004) Influence of fluid viscosity on the hydraulic fracturing mechanism. J Energy Res Technol 126(3):190-200

34. Itasca Consulting Group I (2008) Manual PFC2D (Particle Flow Code), Version 4.0 Users' Guide. Minneapolis, Minnesota, USA: Itasca, Minneapolis

35. Khristianovic S, Zheltov Y (1955) Formation of vertical fractures by means of highly viscous fluids. In: Proceedings of 4th world petroleum congress, Rome, p 579-586

36. Koyama T, Jing L (2007) Effects of model scale and particle size on micro-mechanical properties and failure processes of rocksA particle mechanics approach. Eng Anal Boundary Elem 31(5):458-472

37. Kuhn MR, Sun W, Wang Q (2015) Stress-induced anisotropy in granular materials: fabric, stiffness, and permeability. Acta Geotech 10(4):399-419

38. Latora V, Marchiori M (2003) Economic small-world behavior in weighted networks. Eur Phys J B-Condens Matter 32(2):249-263

39. Li Y, Chen YF, Zhang GJ, Liu Y, Zhou CB (2017) A numerical procedure for modeling the seepage field of water-sealed underground oil and gas storage caverns. Tunn Undergr Space Technol 66:56-63

40. Liu G, Rong G, Peng J, Zhou CB (2015) Numerical simulation on undrained triaxial behavior of saturated soil by a fluid coupledDEM model. Eng Geol 193:256-266

41. Liu G, Cai M, Huang M (2018) Mechanical properties of brittle rock governed by micro-geometric heterogeneity. Comput Geotech. https://doi.org/10.1016/j.compgeo.2017.11.013

42. Marcus D (2008) Graph theory: a problem oriented approach. Mathematical Association of America, Washington, DC

43. Martin CD (1993) The strength of massive Lac du Bonnet granite around underground openings. Ph.D. thesis, University of Manitoba, Winnipeg, Canada

44. Na S, Sun W (2018) Computational thermomechanics of crystalline rock, part I: a combined multi-phase-field/crystal plasticity approach for single crystal simulations. Comput Methods Appl Mech Eng. https://doi.org/10.1016/j.cma.2017.12.022

45. Nguyen VP, Lian H, Rabczuk T, Bordas S (2017) Modelling hydraulic fractures in porous media using flow cohesive interface elements. Eng Geol 225:68-82

46. Nordgren RP (1972) Propagation of a vertical hydraulic fracture. Soc Petrol Eng J 12(04):306-314

47. Ouchi H, Katiyar A, York J, Foster JT, Sharma MM (2015) A fully coupled porous flow and geomechanics model for fluid driven cracks: a peridynamics approach. Comput Mech 55(3):561-576

48. Park CH, Bobet A (2009) Crack coalescence in specimens with open and closed flaws: A comparison. Int J Rock Mech Min Sci 46(5):819-829

49. Park $\mathrm{CH}$, Bobet A (2010) Crack initiation, propagation and coalescence from frictional flaws in uniaxial compression. Eng Fract Mech 77(14):2727-2748

50. Peng J, Wong LNY, Teh CI (2017) Influence of grain size heterogeneity on strength and microcracking behavior of crystalline rocks. J Geophysical Res Solid Earth 122(2):1054-1073

51. Perkins TK, Kern LR (1961) Widths of hydraulic fractures. J Petrol Technol 13(09):937-949

52. Potyondy DO (2010) A grain-based model for rock: approaching the true microstructure. In: Proceedings of rock mechanics in the Nordic Countries
53. Potyondy D (2012) A flat-jointed bonded-particle material for hard rock. In: 46th US Rock mechanics/geomechanics symposium: American Rock Mechanics Association

54. Potyondy DO, Cundall PA (2004) A bonded-particle model for rock. Int J Rock Mech Min Sci 41(8):1329-1364

55. Reyes O, Einstein HH (1991) Failure mechanisms of fractured rock-a fracture coalescence model. In: 7th ISRM Congress: International Society for Rock Mechanics

56. Rong G, Liu G, Hou D, Zhou CB (2013) Effect of particle shape on mechanical behaviors of rocks: a numerical study using clumped particle model. Sci World J. https://doi.org/10.1155/ 2013/589215

57. Rong G, Peng J, Wang X, Liu G, Hou D (2013) Formation mechanism of deep cracks in the left bank slope of Jinping-i hydropower station. Disaster Adv 6(3):4-11

58. Rummel F (1987) Fracture mechanics approach to hydraulic fracturing stress measurements. Academic Press London, Fracture mechanics of rock, pp 217-239

59. Salimzadeh S, Khalili N (2011) Coupling reservoir simulation in naturally fractured reservoir: Implicit versus explicit formulation. In: International Association for Computer Methods and Advances in Geomechanics (IACMAG 13th), Melbourne, Australia, pp 25-30

60. Salimzadeh S, Khalili N (2015) A three-phase XFEM model for hydraulic fracturing with cohesive crack propagation. Comput Geotech 69:82-92

61. Satake M (1992) A discrete-mechanical approach to granular materials. Int J Eng Sci 30(10):1525-1533

62. Shimizu H, Murata S, Ishida T (2011) The distinct element analysis for hydraulic fracturing in hard rock considering fluid viscosity and particle size distribution. Int J Rock Mech Min Sci 48(5):712-727

63. Souley M, Homand F, Pepa S, Hoxha D (2001) Damage-induced permeability changes in granite: a case example at the URL in Canada. Int J Rock Mech Min Sci 38(2):297-310

64. Spence DA, Sharp P (1985) Self-similar solutions for elastohydrodynamic cavity flow. Proc R Soc Lond A Math Phys Eng Sci 400:289-313

65. Sun W (2015) A stabilized finite element formulation for monolithic thermo-hydro-mechanical simulations at finite strain. Int J Numer Meth Eng 103(11):798-839

66. Sun W, Andrade JE, Rudnicki JW, Eichhubl P (2011) Connecting microstructural attributes and permeability from 3D tomographic images of in situ shear-enhanced compaction bands using multiscale computations. Geophys Res Lett 38(10):L10302. https:// doi.org/10.1029/2011GL047683

67. Sun W, Andrade JE, Rudnicki JW (2011) Multiscale method for characterization of porous microstructures and their impact on macroscopic effective permeability. Int $J$ Numer Meth Eng 88(12):1260-1279

68. Sun W, Chen Q, Ostien JT (2014) Modeling the hydro-mechanical responses of strip and circular punch loadings on water-saturated collapsible geomaterials. Acta Geotech 9(5):903-934

69. Thallak S, Rothenburg L, Dusseault M et al (1991) Simulation of multiple hydraulic fractures in a discrete element system. In: The 32nd US symposium on rock mechanics (USRMS): American Rock Mechanics Association

70. Valentini L, Perugini D, Poli G (2007) The, "small-world" topology of rock fracture networks. Physica A 377(1):323-328

71. Valentini L, Perugini D, Poli G (2007) The 'small-world' nature of fracture/conduit networks: possible implications for disequilibrium transport of magmas beneath mid-ocean ridges. J Volcanol Geoth Res 159(4):355-365

72. Vogel HJ, Tölke J, Schulz VP, Krafczyk M, Roth K (2005) Comparison of a lattice-Boltzmann model, a full-morphology 
model, and a pore network model for determining capillary pressure-saturation relationships. Vadose Zone J 4(2):380-388

73. Wang K, Sun W (2017) A unified variational eigen-erosion framework for interacting brittle fractures and compaction bands in fluid-infiltrating porous media. Comput Methods Appl Mech Eng 318(1):1-32

74. Wang K, Sun W (2018) A multiscale multi-permeability poroplasticity model linked by recursive homogenizations and deep learning. Comput Methods Appl Mech Eng 334:337-380

75. Wang SY, Sun L, Au ASK, Yang TH, Tang CA (2009) 2Dnumerical analysis of hydraulic fracturing in heterogeneous geomaterials. Constr Build Mater 23(6):2196-2206

76. Wang T, Zhou W, Chen J, Xiao X, Li Y, Zhao X (2014) Simulation of hydraulic fracturing using particle flow method and application in a coal mine. Int $\mathrm{J}$ Coal Geol 121:1-13

77. Wong LNY, Einstein HH (2009) Crack coalescence in molded gypsum and Carrara marble: part 1. Macroscopic observations and interpretation. Rock Mech Rock Eng 42(3):475-511
78. Wong LNY, Einstein HH (2009) Crack coalescence in molded gypsum and Carrara marble: part 2-microscopic observations and interpretation. Rock Mech Rock Eng 42(3):513-545

79. Zhang X, Jeffrey RG (2012) Fluid-driven multiple fracture growth from a permeable bedding plane intersected by an ascending hydraulic fracture. J Geophys Res 117:B12402. https:// doi.org/10.1029/2012jb009609

80. Zhang F, Damjanac B, Huang H (2013) Coupled discrete element modeling of fluid injection into dense granular media. J Geophys Res Solid Earth 118(6):2703-2722

81. Zhuang X, Augarde C, Mathisen K (2012) Fracture modeling using meshless methods and level sets in 3D: framework and modeling. Int J Numer Meth Eng 92(11):969-998

Publisher's Note Springer Nature remains neutral with regard to jurisdictional claims in published maps and institutional affiliations. 